\title{
Making a Quantum Universe: Symmetry and Gravity
}

\author{
Houri Ziaeepour ${ }^{1,2}$ (D) \\ 1 Institut UTINAM, CNRS UMR 6213, Observatoire de Besançon, Université de Franche Compté, \\ 41 bis ave. de l'Observatoire, BP 1615, 25010 Besançon, France; \\ hz@mssl.ucl.ac.uk or houriziaeepour@gmail.com \\ 2 Mullard Space Science Laboratory, University College London, Holmbury St. Mary, Dorking GU5 6NT, UK
}

Received: 05 September 2020; Accepted: 17 October 2020; Published: 23 October 2020

\begin{abstract}
So far, none of attempts to quantize gravity has led to a satisfactory model that not only describe gravity in the realm of a quantum world, but also its relation to elementary particles and other fundamental forces. Here, we outline the preliminary results for a model of quantum universe, in which gravity is fundamentally and by construction quantic. The model is based on three well motivated assumptions with compelling observational and theoretical evidence: quantum mechanics is valid at all scales; quantum systems are described by their symmetries; universe has infinite independent degrees of freedom. The last assumption means that the Hilbert space of the Universe has $S U(N \rightarrow \infty) \cong$ area preserving Diff. $\left(S_{2}\right)$ symmetry, which is parameterized by two angular variables. We show that, in the absence of a background spacetime, this Universe is trivial and static. Nonetheless, quantum fluctuations break the symmetry and divide the Universe to subsystems. When a subsystem is singled out as reference-observer-and another as clock, two more continuous parameters arise, which can be interpreted as distance and time. We identify the classical spacetime with parameter space of the Hilbert space of the Universe. Therefore, its quantization is meaningless. In this view, the Einstein equation presents the projection of quantum dynamics in the Hilbert space into its parameter space. Finite dimensional symmetries of elementary particles emerge as a consequence of symmetry breaking when the Universe is divided to subsystems/particles, without having any implication for the infinite dimensional symmetry and its associated interaction-percived as gravity. This explains why gravity is a universal force.
\end{abstract}

Keywords: quantum gravity; quantum mechanics; symmetry; quantum cosmology

\section{Introduction and Summary of Results}

More than a century after the discovery of general relativity and description of gravitational force as the modification of spacetime geometry by matter and energy, we still lack a convincing model for explaining these processes in the framework of quantum mechanics. Appendix A briefly reviews the history of efforts for finding a consistent Quantum Gravity (QGR) model. Despite tremendous effort of generations of scientists, none of proposed models presently seem fully satisfactory.

Quantization of gravity is inevitable. Examples of inconsistencies in a universe where matter is ruled by quantum mechanics but gravity is classical are well known [1,2]. In addition, in [2], it is argued that there must be an inherent relation between gravity and quantum mechanics. Otherwise, the universality of Planck constant $\hbar$ as quantization scale cannot be explained. ${ }^{1}$ Aside from these arguments, the fact that there is no fundamental mass/energy scale in quantum mechanics means that it has to have a close relation with gravity that provides a dimensionful fundamental constant, namely

1 Nonetheless, Ref. [3] advocates a context dependent Planck constant. 
the Newton gravitational constant $G_{N}$ or equivalently the Planck mass $M_{P} \equiv \sqrt{\hbar c / G_{N}}$, where $c$ is the speed of light in vacuum (or equivalently Planck length scale $L_{P} \equiv \hbar / c M_{P}$ ). We should remind that a dimensionful scale does not arise in conformal or scale independent models. Indeed, conformal symmetry is broken by gravity, which provides the only fundamental dimensionful constant to play the role of a ruler and make distance and mass measurements meaningful. ${ }^{2}$

In what concerns the subject of this volume, namely representations of inhomogeneous Lorentz symmetry (called also Poincaré group), they were under special interest since decades ago, hoping that they help formulate gravity as a renormalizable quantum field. The similarity of the compact group of local Lorentz transformations to Yang-Mills gauge symmetry has encouraged quantum gravity models that are based on the first order formulation of general relativity. These models use vierbein formalism and extension of gauge group of elementary particles to accommodate Poincaré group [5,6]. However, Coleman-Madula theorem [7] on S-matrix symmetries-local transformations of interacting fields that asymptotically approach Poincaré symmetry at infinity-invalidates any model in which Poincaré and internal symmetries are not factorized. According to this theorem total symmetry of a grand unification model, including gravity, must be a tensor product of spacetime and internal symmetries. Otherwise, the model must be supersymmetric [8] or VEV of the gauge field should not be flat [9]. However, we know that even if supersymmetry is present at $M_{P}$ scale, it is broken at low energies. Moreover, any violation of Coleman-Madula theorem and Lorentz symmetry at high energies can be convoyed to low energies [10] and violate e.g., equivalence principle and other tested predictions of general relativity [11,12]. For these reasons, modern approaches to the unification of gravity as a gauge field with other interactions consider the two sectors as separate gauge field models. In addition, in these models gravity sector usually has topological action to make the formulation independent of the geometry of underlying spacetime, see e.g., [13-15]. However, like other quantum gravity candidates these models suffer from various issues. The separation of internal and gravitational gauge sectors means that these models are not properly speaking a grand unification. Moreover, similar to other approaches to QGR, these models do not clarify the nature of spacetime, its dimensionality, and relation between gravity and internal symmetries.

In addition to consistency with general relativity, cosmology, and particle physics, a quantum model unifying gravity with other forces is expected to solve well known problems that are related to gravity and spacetime, such as: physical origin of the arrow of time; apparent information loss in black holes; and, UV and IR singularities in Quantum Field Theory (QFT) and general gravity. ${ }^{3}$ There are also other issues that a priori should be addressed by a QGR model, but are less discussed in the literature:

1. Should spacetime be considered as a physical entity similar to quantum fields associated to particles, or rather it presents a configuration space?

General relativity changed spacetime from a rigid entity to a deformable media. However, it does not specify whether spacetime is a physical reality or a property of matter, which ultimately determines its geometry and topology. We remind that in the framework of QFT vacuum is not the empty space of classical physics, see e.g., [16,17]. In particular, in the presence of gravity the naive definition of quantum vacuum is frame dependent. A frame-independent definition exists [18] and it is very far from classical concept of an

2 In addition to $M_{P}$, we need two other fundamental constants to describe physics and cosmology: the Planck constant $\hbar$ and maximum speed of information transfer that experiments show to be the speed of light in classical vacuum. We remind that triplet constants $\left(\hbar, c, M_{P}\right)$ are arbitrary and can take any nonzero positive value. The selection of their values amounts to the definition of a system of units for measuring other physical quantities. In QFT literature usually $\hbar=1$ and $c=1$ are used. In this system of units—called high energy physics units [4] $\hbar$ and $c$ are dimensionless.

3 Some quantum gravity models such as loop quantum gravity emphasize the quantization of gravity alone. However, giving the fact that gravity is a universal force and interacts with matter and other forces, its quantization necessarily has impact on them. Therefore, any quantum gravity only model would be, at best, incomplete. 
empty space. Explicitly or implicitly, some of models reviewed in Appendix A address this question.

2. Is there any relation between matter and spacetime?

In general relativity matter modifies the geometry of spacetime, but the two entities are considered as separate and stand alone. In string theory spacetime and matter fields_compactified internal space-are considered and treated together, and spacetime has a physical reality that is similar to matter. By contrast, many other QGR candidates only concentrate their effort on the quantization of spacetime and gravitational interaction. Matter is usually added as an external ingredient and it does not intertwine in the construction of quantum gravity and spacetime.

3. Why do we perceive the Universe as a three-dimensional (3D) space (plus time) ?

None of extensively studied quantum gravity models discussed in Appendix A answer this question, despite the fact that it is the origin of many troubles for them. For instance, the enormous number of possible models in string theory is due to the inevitable compactification of extra-dimensions to reduce the dimension of space to the observed $3+1$. In background independent models, the dimension of space is a fundamental assumption and essential for many technical aspects of their construction. In particular, the definition of Ashtekar variables [19] for $S U(2) \cong S O(3)$ symmetry and its relation with spin foam description of loop quantum gravity [20] are based on the assumption of a 3D real space. On the other hand, according to holography principle, the maximum amount of information that is containable in a quantum system is proportional to its area rather than volume. If the information is projected and available on the boundary, it is puzzling why we should perceive the volume.

In a previous work [21], we advocated the foundational role of symmetries in quantum mechanics and reformulated its axioms accordingly, see Appendix B for a summary. Of course, the crucial role of symmetries in quantum systems is well known. However, axioms of quantum mechanics à la Dirac and von Neumann consider an abstract Hilbert space and do not specify its relation with symmetries of quantum systems. In addition to symmetries of their classical Lagrangian, Hilbert space of quantum systems represents $S U(N)$ group, called state symmetry, see Appendix C for more details. Transformation of states by this group modifies their coherence, and recently quantification of this property and its usefulness as a resource has become a subject of interest in quantum information theory literature [22,23].

Inspired by these developments, in this work we study a standalone quantum system, which is considered to be the Universe.

\subsection{Summary of the Model and Results}

The model assumes infinite number of independent and simultaneously commuting observables in the Universe, but no background spacetime. Hilbert space $\mathcal{H}_{U}$ of such system represents $S U(N \rightarrow \infty)$ symmetry. However, in absence of a background spacetime, its dynamics is trivial and its Lagrangian is defined on the group manifold of $S U(\infty)$ symmetry. Therefore, states are pure gauge. The vector space of gauge transformations, corresponding to linear transformations of the Hilbert space, is $\mathcal{B}\left[\mathcal{H}_{U}\right] \cong S U(\infty)$. On the other hand, quantum fluctuations break the state symmetry and factorize the Hilbert space to blocks of tensor product of subspaces according to criteria studied in $[24,25]$. For each subsystem, the rest of the Universe plays the role of a background parameterized by three continuous quantities that can be identified with the classical space. Moreover, division of the Universe to subsystems leads to emergence of time and its arrow à la Page \& Wootters [26] or similar methods [27]. We show that the $3+1$ dimensional parameter space is, in general, curved and 
invariant under inhomogeneous Lorentz transformations and its curvature is determined by quantum states of the subsystems. We also comment on the signature of parameter space metric. Based on these observations, we interpret $S U(\infty)$ sector of the model as Quantum Gravity. The finite rank factorized symmetries become local gauge fields acting on a Hilbert space that presents matter fields.

These results demonstrate the importance of the division of Universe to subsystems and the distinction of observer and clock from the rest. Nonetheless, in contrast to the Copenhagen interpretation of quantum mechanics, the absence of observer does not make the model meaningless, but trivial and static. This model answers some of issues raised in questions 1-3 raised earlier. In particular, it clarifies the nature of spacetime and its dimensionality, and provides an explanation for the universality of gravitational force.

A crucial proposal of the model is that what we perceive as classical spacetime is the configuration (parameter) space of its content. In other words, rather than saying particles/objects (such as strings) live in a $3+1$ dimensional space, according to this model we can say that an ensemble of abstract objects with $S U(\infty) \times G$ symmetry look like a $3+1$ dimensional infinite curved spacetime with gravity, where subsystems are fields that represent group $G$ as a local gauge symmetry. Thus, we can completely neglect the geometric interpretation and just consider the Universe as an infinite tensor product. This aspect of the model is similar to the approach of [28]. However, their model is somehow inverse of that studied here. They use tensor product and quantum entanglement to make a symplectic geometry that becomes a continuous curved spacetime when the number of tensor product factors approaches to infinity. The drawback is that symplectic geometries defined by graphs can be embedded in any space of dimension $D \geqslant 2$. Consequently, they cannot explain the dimension of the spacetime.

Axioms and structure of the model is discussed in Section 2. Lagrangian of the system before its division is described in Section 3. Properties of the model after symmetry breaking and division of the Universe are studied in Section 4. Section 5 presents a brief comparison of this model with string and loop quantum gravity. Section 6 presents outlines and prospective for future investigations. Accompanying appendices contain technical details and review of previous results. Appendix A gives a short recount of the history of quantum gravity models. Appendix B summaries the axioms of quantum mechanics in symmetry language. State space and its associated symmetry is reviewed in Appendix C. Properties of $S U(\infty)$ and its representations are summarized in Appendix D and its Cartan decomposition in Appendix E.

\section{An Infinite Quantum Universe}

Our departure point for constructing a quantum universe consists of three well motivated assumptions with compelling observational and theoretical evidence:

I. Quantum mechanics is valid at all scales and applies to every entity, including the Universe as a whole;

II. Any quantum system is described by its symmetries and its Hilbert space represents them;

III. The Universe has an infinite number of independent degrees of freedom.

The last assumption means that the Hilbert space of the Universe $\mathcal{H}_{U}$ is infinite dimensional and represents the group $S U(\infty)$. There is sufficient evidence in favour of such an assumption. For instance, the thermal distribution of photons at IR limit contains an infinite number of quanta with energies approaching zero and there is no minimum energy limit. For this reason, vacuum can be considered to be a superposition of multi-particle states of any type-not just photons-without any limit on their number [18]. In general relativity, there is no upper limit for gravitons wavelength and thereby their number. Of course, one may argue that a lower limit on energy or spacetime volume may exist. Nonetheless, for any practical application the number of subsystems/quanta in the Universe can be considered to approach infinity. Indeed, even in quantum gravity models that assume a symplectic structure for spacetime, such as spin foam/loop quantum gravity and causal sets, there is not a fixed lattice of spacetime and the number of spacetime states is effectively infinite. 
The algebra that is associated to the $S U(\infty)$ coherence (state) symmetry of the above model is defined as ${ }^{4}$ :

$$
\left[\hat{L}_{a}, \hat{L}_{b}\right]=\frac{\hbar}{c M_{P}} f_{a b}^{c} \hat{L}_{c}=L_{P} f_{a b}^{c} \hat{L}_{c}
$$

where operators $\hat{L}_{\alpha} \in \mathcal{B}\left[\mathcal{H}_{U}\right]$ are generators of algebra $s u(\infty)$ and $f_{a b}^{c}$ are its structure coefficients. They are normalized such that the r.h.s. of (1) explicitly depends on the Planck constant $\hbar$. If $\hbar \rightarrow$ 0 , the r.h.s. becomes null, and the algebra becomes abelian and homomorphic to $\otimes^{N \rightarrow \infty} U(1)$ in agreement with the symmetry of configuration space of classical systems, explained in Appendix C. The same happens if $M_{P} \rightarrow \infty$, that is when Planck mass scale is much larger than scale of interest. In both cases $L_{P} \rightarrow 0$. Assuming that $S U(\infty)$ symmetry of the Universe can be associated to gravitational interaction -we will provide more evidence in favour of this claim later-the above limits mean that, in both cases, gravity becomes negligible. ${ }^{5}$

It is well known that $\mathcal{B}\left[\mathcal{H}_{U}\right] \cong S U(\infty) \cong$ area preserving $\operatorname{Diff}\left(S_{2}\right)[29,30]$, where $S_{2}$ is $2 \mathrm{D}$ sphere. In fact, $S U(\infty)$ is homomorphic to area preserving diffeomorphism of any two-dimensional (2D) Riemann surface [31-33]. Therefore, here $S_{2}$ can be any 2D surface, rather than just sphere. This theorem can be heuristically understood as the following: any compact 2D Riemann surface can be obtained from sphere by removing a measure zero set of pairs of points and sticking the rest of the surface pair-by-pair together. Although surfaces with different genus are topologically different, they are homomorphic. This property may be important in the presence of subsystems with singularity, such as black holes, in which part of the parameter space is inaccessible. From now on, we call a 2D surface that its diffeomorphism represents $S U(\infty)$ a diffeo-surface.

Homomorphism between $S U(\infty)$ and $\operatorname{Diff}\left(S_{2}\right)$ makes it possible to expand $\hat{L}_{a}$ 's with respect to spherical harmonic functions, depending on angular coordinates $(\theta, \phi)$ on a sphere. Moreover, owing to the Cartan decomposition, $S U(\infty)$ generators can be described as a tensor product of Pauli matrices [29,34]. In this case, indices in (1) consist of a pair $(l, m) \mid l=0, \cdots, \infty ;-l \leqslant m \leqslant+l$. Appendix E reviews decomposition and indexing of $S U(\infty)$ generators. We continue to use single letters for the indices of generators when there is no need for their explicit description.

The algebra (1) is not enough to make the system quantic and as usual $\hat{L}_{a}$ 's must respect Heisenberg commutation relations:

$$
\left[\hat{L}_{a}, \hat{J}_{b}\right]=-i \delta_{a b} \hbar \text {. }
$$

where $\hat{J}_{a} \in \mathcal{B}\left[\mathcal{H}_{U}^{*}\right]$ is the dual of $\hat{L}_{a}$ and $\mathcal{H}_{U}^{*}$ is the dual Hilbert space of the Universe. As there is a one-to-one correspondence between $\hat{L}^{\prime} \mathrm{s}$ and $\hat{J}^{\prime} \mathrm{s}$, they satisfy the same algebra, represent the same symmetry group, namely $S U(\infty)$, and they have their own expansion to spherical harmonics. Owing to $S U(\infty) \cong \operatorname{Diff}\left(S_{2}\right)$, vectors of the Hilbert space are differentiable complex functions of angular coordinates $(\theta, \phi)$. Thus, spherical harmonic functions constitute an orthogonal basis for $\mathcal{H}_{U}$. The Cartan subalgebra of $\mathcal{B}\left[\mathcal{H}_{U}\right] \cong S U(\infty)$ is also infinite dimensional.

The quantum Universe defined here is static, because there is no background space or time in the model. Nonetheless, in Section 4, we show that continuous degrees of freedom similar to space and time naturally arise when the Universe is divided to subsystems. The short argument goes as the following:

We assume that eigen states of the Hilbert space of the Universe are not abstract objects and physically exist. This assumption is supported by the fact that in Standard Model (SM) of particle physics states that constitute a basis for its Hilbert space and for its space of linear transformations are indeed observed particles (fields). Consequently, taking into account the assumption that $\mathcal{H}_{U}$ is infinite

4 In this work, all vector spaces and algebras are defined on complex number field $\mathbb{C}$, unless explicitly mentioned otherwise. Although in (1) we show the dimensional scale $\hbar / M_{P}$ in the definition of operators and their algebra, for the sake of convenience in the rest of this work, we include it in the operators, except when its explicit presentation is necessary for the discussion. 
dimensional, we conclude that the Universe must consist of infinite number of particles/subsystems. Although subsystems may have some common properties, which make them indistinguishable from each others, there are many other distinguishable aspects, which discriminate them from each others. This statement is in agreement with the corollary presented in Appendix B regarding the divisibility of a quantum Universe, derived from axioms of quantum mechanics. Thus, this conclusion is independent of details of the model.

Notice that, without the assumption about physical existence of eigen states, an infinite dimensional Hilbert space does not necessarily mean Universe must be infinitely divisible. Hilbert space of many quantum systems have an infinite number of states. However, they do not necessarily occur in each instance (copy) of the system. The case of a Universe is different, because, by definition, there is only one copy of it. Therefore, every eigen state of a complete basis of its Hilbert space must physically exist. Otherwise, it can be completely discarded.

In the next sections, we make this argument more rigorous and explain how it can lead to a $3+1$ dimensional spacetime and internal gauge symmetry of elementary particles. We begin with constructing a Lagrangian for this static model and show that it is trivial.

\section{Lagrangian of the Universe}

Although the infinite dimensional Universe described in the previous section is static, it has to satisfy constraints imposed by symmetries associated to it. They are analogous to constraints imposed on systems in thermodynamic equilibrium. Although there is no time variation in such systems, a priori small perturbations occur, for instance, by absorption and emission of energy. They must be in balance with each others, otherwise the system would lose its equilibrium. Therefore, it is useful to define a Lagrangian that quantifies these constraints. In the case of present model, the Lagrangian should quantify $S U(\infty)$ symmetry and its representation by $\mathcal{H}_{U}$.

Lagrangian of a system must be invariant under transformations of fields by application of members of its symmetry group. As there is no background spacetime in this model, the most appealing candidate is a Lagrangian similar to Yang-Mills, but without a kinetic term. In such a situation, the only available quantities are invariants of the symmetry group:

$$
\mathcal{L}_{U}=\int d^{2} \Omega \sqrt{\left|g^{(2)}\right|}\left[\frac{1}{2} \sum_{a, b} L_{a}^{*}(\theta, \phi) L_{b}(\theta, \phi) \operatorname{tr}\left(\hat{L}_{a} \hat{L}_{b}\right)+\frac{1}{2} \sum_{a} L_{a} \operatorname{tr}\left(\hat{L}_{a} \rho(\theta, \phi)\right)\right], \quad d^{2} \Omega \equiv d(\cos \theta) d \phi
$$

where $g$ is the determinant of 2D metric of the diffeo-surface. If we use description (A5) for $\hat{L}$ operators, $a=b=1$. If we use (A12) expression, $a, b=(l, m), l=0, \cdots, \infty ;-m \leqslant l \leqslant+m$. The latter case explicitly demonstrates the Cartan decomposition of $S U(\infty)$ to $S U(2)$ factors, as described in Appendix E. Notice that $(\theta, \phi)$ are internal variables [30], reflecting the fact that vectors of the Hilbert space representing $S U(\infty)$ are functions on a 2D Riemann surface. For the same reason, in contrast to usual Lagrangians in QFT, there is no term containing derivatives with respect to these parameters in $\mathcal{L}_{U}$. If we use differential representation of $\hat{L}_{l m}$ defined in (A5) and apply it to amplitudes $L_{l m}(\theta, \phi)$, the first term in the Lagrangian will depend on the partial derivatives of amplitudes, just like in the QFT. However, it is straightforward to see that derivatives with respect to $\cos \theta$ and $\phi$ will have different amplitudes and, thereby, the kinetic term will be unconventional and non-covariant, unless we consider amplitudes $L_{l m}(\theta, \phi)$ as functions of the metric of a deformed sphere. This is the explicit demonstration of $S U(\infty) \cong \operatorname{Diff}\left(S_{2}\right)$ invariance of this Lagrangian.

Generators $\hat{T}_{a}, \hat{T}_{b} \in S U(N), \forall N$ can be normalized, such that $\operatorname{tr}\left(T_{a} T_{b}\right) \propto \delta_{a b}$, see e.g., [29]. In analogy with field strength in Yang-Mills theories, the function $L_{a}(\theta, \phi)$ can be interpreted as the amplitude of the contribution of operator $\hat{L}_{a}$ in the dynamics of the Universe. Due to global $U(1)$ symmetry of operators applied to a quantum state, $L_{a}{ }^{\prime} \mathrm{s}$ are, in general, complex. On the other hand, when considering the Cartan decomposition of $S U(\infty)$ to tensor product of $S U(2)$ factors and the fact that $\sigma^{\dagger}=(\sigma *)^{t}=\sigma$, we conclude that $\hat{L}_{a}^{\dagger}=\hat{L}_{a}$, Similar to QFT, one can use $\mathcal{L}_{U}$ to define a path 
integral. In the absence of time, the path integral presents the excursion of states in the Hilbert space by successive application of $\hat{L}_{a}$ operators. Nonetheless, owing to $S U(\infty)$ symmetry, variation of states is equivalent to gauge transformation and non-measurable.

The analogy of $\mathcal{L}_{U}$ with Yang-Mills theory has interesting consequences. For instance, differential representation of $\hat{L}_{l m}$ defined in (A5) can be written as $\hat{L}_{l m}=\sqrt{\mid g^{(2)}} \mid \epsilon^{\mu v}\left(\partial_{\mu} Y_{l m}\right) \partial_{\nu}$. In classical limit, one can consider that $\hat{L}_{l m}$ acts on the field amplitude $L_{l m}$ and the first term in the integrand of Lagrangian $\mathcal{L}_{U}$ can be arranged, such that it becomes proportional to Ricci scalar $R^{(2)}$. As the geometry of $2 \mathrm{D}$ diffeo-surface is arbitrary, for each set of $L_{l m}$ the metric $g_{\mu \nu}$ can be chosen, such that $L_{l m}$ dependent part of the integrand becomes proportional to Ricci scalar for that metric. Thus, in classical limit the first term is topological. ${ }^{6}$ We could arrive to this conclusion inversely. Because $S U(\infty) \cong \operatorname{Diff}\left(S_{2}\right)$, in the classical limit the Lagrangian should be the same as Einstein gravity in a static 2D curve space. Thus, the first term in (3) can be replaced by $\int d^{2} \Omega \sqrt{\left|g^{(2)}\right|} R^{(2)}$. Then, the definition of $\hat{L}_{l m}$ operators in (A5) and amplitudes $L_{l m}$ can be used to write $R^{(2)}$ with respect to $\hat{L}_{l m}$ and relate metric and connection of the 2D surface to amplitudes $€_{l m}$. We leave a detailed demonstration of these relations to a future work. The relation between gauge field term in $\mathcal{L}_{U}$ and Riemann curvature in classical limit is crucial for interpretation of this term as gravity when the Universe is divided to subsystems.

Notice that, in both representations of $S U(\infty)$, namely Cartan decomposition to tensor product of $S U(2)$ factors and diffeomorphism of 2D surfaces, angular coordinates $\theta$ and $\phi$ play the role of parameters that identify/index the members of the symmetry group. Consequently, their quantization is meaningless. This is consistent with interpretation of Einstein equation as an equation of state [35]. Presuming the physical reality of Hilbert space and operators applied to it, as discussed in the previous section and in Appendix $C$, we can interpret $L_{l m}$ as intensity of force mediator particles related to the symmetry represented by operators $\hat{L}_{l m}$, and $\rho$ in the second term of the Lagrangian $\mathcal{L}_{U}$ as density matrix of matter.

Although $\mathcal{L}_{U}$ is static, we can apply a variational principle with respect to amplitudes to obtain field equations and find equilibrium values of $L_{l m}$ and $\rho$. However, it is easily seen that solutions of these equations are trivial. At equilibrium $L_{l m} \rightarrow 0$ and $\rho_{l m} \rightarrow 0$, see Appendix E.2 for the details. Because $S U(\infty)^{n} \cong S U(\infty) \forall n$, this solution has properties of a frame independent vacuum of a many-particle Universe defined by using coherent states [18]. Their similarity implicitly implies that the Universe is divisible and consists of infinite number of particles/subsystems interacting through mediator particles of $S U(\infty)$ force, which is the action of $\hat{L}_{l m}$. We investigate this conclusion in more details in the next section.

\section{Division to Subsystems}

There are many ways to see that the quantum vacuum (equilibrium) solution of a Universe with $\mathcal{L}_{U}$ Lagrangian (3) is not stable. Of course there are quantum fluctuations. They are nothing else than random application of $\hat{L}_{l m}$ operators, in other words random scattering of force mediator quanta by matter. They project the Hilbert space to itself. However, owing to $S U(\infty)$ symmetry of Lagrangian, states are globally equivalent and the Universe maintain its equilibrium. Nonetheless, locally states are different and they do not respond to $\hat{L}_{l m}$ in the same manner. Here, locality means restriction of Lagrangian and projections to a subspace of the Hilbert space [25]. As state space is homomorphic to the space of smooth functions on the sphere $f(\theta, \phi)$, the restriction of transformations to a subspace is equivalent to a local deformation of the diffeo-surface. Moreover, the difference between structure coefficients of $S U(\infty)$ can be used to define a locality or closeness among operators that belong to

6 We remind that $\int_{\mathcal{M}} d^{2} \Omega \sqrt{\left|g^{(2)}\right|} R^{(2)}=4 \pi \chi(\mathcal{M})$, where $\chi$ is the Euler characteristic of the compact Riemann 2D surface $\mathcal{M}$. Moreover, Ricci scalar alone does not determine Riemann curvature tensor $R_{\mu v}$ and only provides one constraint for three independent components of the metric tensor. 
$\mathcal{B}\left[\mathcal{H}_{U}\right]$. These observations are additional evidence to the argument given at the end of Section 3 in favour of the divisibility of the quantum Universe introduced in Section 2 to multi-particle/subsystems.

A quantum system that is divisible to separate and distinguishable subsystems ${ }^{7}$ must fulfill 3 conditions [24]:

- There must exist sets of operators $\left\{A_{i}\right\} \subset \mathcal{B}[\mathcal{H}]$ such that $\forall \bar{a} \in\left\{A_{i}\right\}$ and $\forall \bar{b} \in\left\{A_{j}\right\}$, and $i \neq$ $j,[\bar{a}, \bar{b}]=0$;

- Operators in each set $\left\{A_{i}\right\}$ must be local ${ }^{8}$;

- $\left\{A_{i}\right\}^{\prime}$ s must be complementarity, which is $\otimes_{i}\left\{A_{i}\right\} \cong \operatorname{End}(\mathcal{B}[\mathcal{H}])$.

The most trivial way of fulfilling these conditions is a reducible representation of symmetries by $\mathcal{B}[\mathcal{H}]$. In the case of $\mathcal{B}\left[\mathcal{H}_{U}\right] \cong S U(\infty)$, as:

$$
S U(\infty)^{n} \cong S U(\infty) \forall n
$$

the above condition can be easily realized. Moreover, instabilities, quantum correlations, and entanglement may create local symmetries among groups of states and/or operators. There are many examples of such grouping and induced symmetries in many-body systems, see e.g., [36] for a review. A hallmark of induced symmetry by quantum correlations is the formation of anyon quasi-particles having non-abelian symmetry in the fractional quantum Hall effect [37]. On the other hand, there is only one state in the infinite dimensional Hilbert space, in which all pointer states have the same probability, namely the maximally coherent state defined in (A2). Even if a many-body system begins in such a maximally symmetric state, quantum fluctuations rapidly change it to a less coherent and more asymmetric one. In addition, due to (4), irreducible representations of $S U(\infty)$ are partially entangled [25] and there is high probability of clustering of subspaces in a randomly selected state.

Lets assume that such groupings indeed have occurred in the early Universe and they continue to occur at Planck scale. They provide the necessary conditions for division of the Universe to parts or particles with $S U(\infty) \times G \cong S U(\infty)$ as their symmetry. The local symmetry $G$ is assumed to be a compact Lie group of finite rank and respected by all subsystems. Although different subsystems may have different internal symmetries, without a lack of generality, we can assume that $G$ is their tensor product, but some species of particles/subsystems are in singlet representation of some of the component groups.

As the rank of $G$ is assumed to be finite, complementarity condition dictates that the number of subsystems must be infinite to account for the infinite rank of $\mathcal{H}_{U}$. If states are in a finite dimensional representations of $G$, at least one of the representations must have infinite multiplicity and their Hilbert space would be infinite dimensional. Thus, despite the division of $\mathcal{B}\left[\mathcal{H}_{U}\right], S U(\infty)$ remains a symmetry of subsystems and $\left\{A_{i}\right\} \subset \mathcal{B}\left[\mathcal{H}_{i}\right] \cong S U(\infty)$, where $\mathcal{H}_{i}$ is the Hilbert space of subsystem $i$. Clustering of states and subsystems are usually the hallmark of strong interaction and quantum correlation [36]. Therefore, the interaction of subsystems through internal $G$ symmetry is expected to be stronger than through $S U(\infty)$, thereby the weak gravitation conjecture [38] is satisfied.

We could also formulate the above Universe in a bottom-up manner. Consider the ensemble of infinite number of quantum systems-particles—each having finite symmetry $G$ and coherently mixed with each others. Their ensemble generates a Universe with $S U(\infty) \times G \cong S U(\infty)$ as symmetry represented by its Hilbert space. Therefore, top-down or bottom-up approaches to an infinitely

7 In statistical quantum or classical mechanics distinguishability of particles usually means being able to say, for instance, whether it was particle 1 or particle 2 which was observed. Here by distinguishability we mean whether a particle/subsystem can be experimentally detected, i.e., through application of $\hat{L}_{l m}$ to a subspace of parameter space and identified in isolation from other subsystems or the rest of the Universe.

8 This condition is defined for quantum systems in a background spacetime. In the present model there is not such a background. Nonetheless, as explained earlier, locality on the diffeo-surface can be projected to $\mathcal{B}\left[\mathcal{H}_{U}\right]$. 
divisible Universe give the same result. The bottom-up view helps to better understand the origin of $S U(\infty)$ symmetry. It shows that, for each subsystem, it is the presence of other infinite number of subsystems and its own interaction with them that is seen as a $S U(\infty)$ symmetry.

\subsection{Properties of an Infinitely Divided Quantum Universe}

The division of the Universe to subsystems has several consequences. First of all, the global $U(1)$ symmetry of $\mathcal{H}_{U}$ becomes local, because Hilbert spaces of subsystems $\mathcal{H}_{i}, \forall i$, where index $i$ runs over all subsystems, acquire their own phase symmetry. Therefore, we expect that there is at least one unbroken $U(1)$ local—gauge-symmetry in nature. It may be identified as $U(1)$ symmetry of the Standard Model. From now on, we include this $U(1)$ to the internal symmetry of subsystems G. Additionally, the infinite number of subsystems in the Universe means that each of them has its own representation of $S U(\infty)$ symmetry. However, these representations are not isolated and are part of the $S U(\infty)$ symmetry of the whole Universe. This property is similar to finite intervals on a line, which are homomorphic to $R^{(1)}$ and, at the same time, part of it and have the same algebra. Therefore, the memory of being part of the whole Universe is not washed out with the division to subsystems. Otherwise, according to the corollary discussed in Appendix B subsystems could be considered as separate and isolated universes.

The area of diffeo-surface is irrelevant when only one $S U(\infty)$ is considered. However, it becomes relevant and observable when it is compared with its counterparts for other subsystems. More precisely, homomorphism between Hilbert spaces of two subsystems $s$ and $s^{\prime}$ defined as:

$$
\mathcal{R}_{s s^{\prime}}: \mathcal{H}_{s} \rightarrow \mathcal{H}_{s^{\prime}}
$$

can be considered as an additional parameter that is necessary for their identification and indexing. A more qualitative description of how a third continuous parameter emerges from division of Universe to subsystem is given in the next subsection.

\subsection{Parameterization of Subsystems}

There are various ways to see that the division of the Universe to subsystems defined in Section 2 induces a new continuous parameter. As discussed in the previous subsection, each subsystem represents $S U(\infty) \times G$. When $S U(\infty)$ representation of different subsystems are compared, e.g., through a morphism, the radius of diffeo-surface becomes relevant, because different radius means different area. This dependence allows for classifying subsystems according to a size scale. More precisely, in the definition of $\hat{L}_{l m}$ in (A5), $Y_{l m} \propto r l$, where $r$ is the distance to centre in spherical coordinates when the 2D surface is embedded in $R^{(3)}$. If we factorize $r$-dependence part of $Y_{l m}$, the algebra of $\hat{L}_{l m}$ defined in (A4) becomes:

$$
\left.\left[\hat{L}_{l m}, \hat{L}_{l^{\prime} m^{\prime}}\right]\right|_{r=1}=\left.r^{l^{\prime \prime}-l^{\prime}-l} f_{l m, l^{\prime} m^{\prime}}^{l^{\prime \prime} m^{\prime \prime}} \hat{L}_{l^{\prime \prime} m^{\prime \prime}}\right|_{r=1}
$$

where all $\hat{L}_{l m}$ operators are defined for $r=1$ (in an arbitrary unit). Equation (6) shows that $r$ can be interpreted as a coupling that quantifies the strength of correlation between $\hat{L}_{l m}$ operators. Moreover, due to homomorphism (4), $\hat{L}_{l m}$ 's of subsystems are part of $\hat{L}_{l m}$ 's of the full system. Consequently, subsystems are never completely isolated and they interact through an algebra similar to (6), but their $r$ factors can be different:

$$
\left.\left[\hat{L}_{l m}^{(r)}, \hat{L}_{l^{\prime} m^{\prime}}^{\left(r^{\prime}\right)}\right]\right|_{r=1}=\left.r^{\prime \prime l^{\prime \prime}} r^{{ }^{-l^{\prime}}} r^{-l} f_{l m, l^{\prime} m^{\prime}}^{l^{\prime \prime} m^{\prime \prime}} \hat{L}_{l^{\prime \prime} m^{\prime \prime}}^{r^{\prime \prime}}\right|_{r=1}
$$

where $r$ indices on $\hat{L}_{l m}$ operators are added to indicate that they may belong to different subsystems. Nonetheless, the algebra remains the same, because operators $\hat{L}_{l m}$ belong, at the same time, to the global 
$S U(\infty)$. On the other hand, the nonlocality of this algebra in the point of view of subsystems should induce a dependence on derivative with respect to parameters when infinitesimal transformations are considered, e.g., in the Lagrangian. Specifically, we expect a relation between $r^{\prime \prime}$ and $\left(r, r^{\prime}\right)$, determined by homomorphism (5). In the infinitesimal limit, the r.h.s. of (7) becomes Lie derivative of $\hat{L}_{l m}$ in the direction of $\hat{L}_{l^{\prime} m^{\prime}}$ in the manifold that is defined by parameters $(r, \theta, \phi, t)$, where the last parameter is time with respect to an observer, as described in the next subsection.

In summary, after the division of the Universe to subsystems, their $S U(\infty)$ symmetries are indexed by angular parameters $(\theta, \phi)$ and an additional continuous parameter $r=(0, \infty)$. They share the algebra of global $S U(\infty)$, but acquire a new index and, in this sense, their algebra becomes nonlocal. Notably, in the infinitesimal limit the algebra can be considered as the Lie derivative of $\hat{L}_{a} \in \mathcal{B}[\mathcal{H}]$ operators on the manifold of parameter space $(r$, thet $a, \phi, t)$. Differential properties of the model need more investigation and will be reported elsewhere.

Finally, we can define a conjugate set of parameters for the dual Hilbert space $\mathcal{H}_{U}^{*}$ and dual operators $\hat{J}_{a}$ defined in (2). Therefore, in contrast to some quantum gravity candidates, this model does not have a preference for position or momentum spaces.

\subsection{Clocks and Dynamics}

The last step for construction of a dynamical quantum Universe is the introduction of a clock by using comparison between variation of states of two subsystems, tagged as system and clock, under the application of operators $\hat{L}_{\alpha} \in S U(\infty) \times G$ by a third subsystem, tagged as observer, who plays the role of a reference. The necessity of an observer/reference is consistent with the foundation of quantum mechanics, as described in [21]. In the context of the present model, this discrimination can be understood as the following: although the global $S U(\infty)$ symmetry means that any variation of full state by application of $\hat{L}_{\alpha}$ is a gauge transformation, a variation of subsystems with respect to each others is meaningful and can be quantified.

The technical details of introducing a clock and relative time in quantum mechanics are intensively studied in the literature, see e.g., [27] for a review and proof of the equivalence of different approaches. Here, we describe this procedure through an example. Consider the application of operators $\hat{L}_{c} \in \mathcal{B}\left[\mathcal{H}_{C}\right]$ and $\hat{L}_{s} \in \mathcal{B}\left[\mathcal{H}_{s}\right]$ to two subsystems, called clock and system, respectively, such that:

$$
\hat{L}_{c} \rho_{c} \hat{L}_{c}^{\dagger}=\rho_{c}+d \rho_{c}=d \rho_{c}^{\prime}, \quad \hat{L}_{s} \rho_{s} \hat{L}_{s}^{\dagger}=\rho_{s}+d \rho_{s}=d \rho_{s}^{\prime}
$$

Because these operations are local and restricted to subsystems, they are not gauged out. One way of associating a c-number quantity to these variations is to define parameter $t$, such that, for instance, $d t \equiv\left|\operatorname{tr}\left(\rho_{c}^{\prime} \hat{O}_{c}\right)-\operatorname{tr}\left(\rho_{c} \hat{O}_{c}\right)\right|$, where $\hat{O}_{c}$ is an observable of the clock subsystem. This quantity is positive and, by definition, incremental. The Hamiltonian operator of the system $H_{s} \in \mathcal{B}\left[\mathcal{H}_{s}\right]$ according to this clock would be an operator for which $d \rho_{s} / d t=-i / \hbar\left[H_{s}, \rho_{s}\right]$.

More generally, defining a clock is equivalent to comparing excursion path of two subsystems in their respective Hilbert space under successive application of $\hat{L}_{c}$ and $\hat{L}_{s}$ to them, respectively. The arrow of time arises because through the common $S U(\infty)$ symmetry any operation-even a local one-is communicated to the whole Universe. Thus, inverting the arrow of time amounts to performing an inverse operation on all subsystems, which is extremely difficult. Therefore, although the dynamical equation of one system may be locally symmetric with respect to time reversal, due to global effect of every operation, its effect cannot be easily reversed.

\subsection{Geometry of Parameter Space}

The final stride of time definition brings the dimension of continuous parameter space necessary for describing states and dynamics of an infinite dimensional divisible Universe to $3+1$, namely 
$(r, \theta, \phi, t) \cdot{ }^{9}$ Although these parameters arise from different properties of the Universe, namely $(\theta, \phi)$ from $S U(\infty)$ symmetry, $r$ from division to infinite number of subsystems, and $t$ from their relative variation, they are mixed through the global $S U(\infty)$ symmetry, arbitrariness of the choice of reference frame and clock, and quantum superposition of states. Therefore, geometry of the parameter space is $R^{(3+1)}$.

The 2D parameter space of the whole Universe is, by definition, diffeomorphism invariant, as it is the representation of $S U(\infty)$. However, at this stage it is not clear whether the subdivided Universe is rigid, that is only invariant only under global frame transformations of the $(3+1) \mathrm{D}$ parameter space, or deformable and invariant under its diffeomorphism. Here we show that it is indeed diffeomorphism invariant. Moreover, its geometry is determined by states of subsystems. ${ }^{10}$

Consider a set of 2D diffeo-surfaces representing $S U(\infty)$ symmetries of subsystem. These diffeomorphism can be obtained from application of $\hat{L}_{l m} \in S U(\infty)$ operators to vacuum state of each subsystem, considered to be a sphere. They are smooth functions of parameters $(r, \theta, \phi)$ and can be identified with states of the subsystems, which are also smooth functions of the same parameters. After ordering these surfaces-for instance, according to their average distances ${ }^{11}$-and defining a projection between neighbours ${ }^{12}$, such that if on $i$ th surface the point $\left(\theta_{i}, \phi_{i}, r_{i}\right)$ is projected to $\left(\theta_{i+1}, \phi_{i+1}, r_{i+1}\right)$ on $(i+1)$ th surface, the distance in $R^{(3)}$ between points in an infinitesimal surface $\Delta \Omega_{i}<\epsilon^{2}$ containg $\left(\theta_{i}, \phi_{i}, r_{i}\right)$ and infinitesimal surface $\Delta \Omega_{i+1}<\epsilon^{\prime 2}$ containg $\left(\theta_{i+1}, \phi_{i+1}, r_{i+1}\right)$ approaches zero if $\epsilon, \epsilon^{\prime} \rightarrow 0$. The path connecting closest points on $\Delta \Omega_{i}$ and $\Delta \Omega_{i+1}$ defines an orthogonal direction in a deformed $S_{2} \times R^{(1) \cong} R^{(3)}$ and the Riemann curvature of this space can be determined from sectional curvature. Therefore, parameter space (or equivalently Hilbert space) is curved. Moreover, as the projection between $\Delta \Omega_{i}$ and $\Delta \Omega_{i+1}$ used for this demonstration is arbitrary, we conclude that the parameter space is not rigid and its diffeomorphism does not change the physics. The same procedure can be applied when a clock is chosen. Therefore, the above conclusions apply to the full $(3+1) D$ parameter space of the subdivided Universe.

Finally, from homomorphism between diffeo-surfaces and states of subsystems, we conclude that $(3+1) D$ classical spacetime can be interpreted as parameter space of the Hilbert space of subsystems of the Universe, and gravity as the interaction that is associated to $S U(\infty)$ symmetry.

\subsection{Metric Signature}

Up to now we indicated the dimension of spacetime-parameter space of $S U(\infty)$ symmetry-after subdivision of the Universe as $3+1$. This implicitly means that we have considered a Lorentzian metric with negative signature. In special and general relativity, the signature of metric is dictated by observation of the constant speed of light in classical vacuum. Indeed, diffeomorphism invariance, Einstein equation, and interpretation of gravity as curvature of spacetime are independent of signature of the spacetime metric.

9 Evidently, in addition to $3+1$ external parameters each subsystem represents the internal symmetry $G$, where its representations have their own parameters.

10 Notice that even in classical general relativity diffeomorphism and relation between geometry and state of matter are independent concepts. In particular, Einstein equation is not the only possible relation and a priori other diffeomorphism invariant relations between geometry and matter are allowed-but constrained by experiments.

11 More generally, any measure of difference between states, such as Fubini-Study metric or fidelity can be used to order states. As Hilbert spaces of quantum systems with $S U(\infty)$ symmetry consist of continuous functions, we can use usual analytical tools for defining a distance. However, we should not forget that functions are vectors of a Hilbert space. Moreover, Hilbert space vectors are, in general, complex functions and each projection between diffeo-surfaces corresponds to two projection in the Hilbert space, one for real part and one for imaginary part of vectors.

12 This projection is isomorphic to a homomorphism between $\mathcal{B}\left[\mathcal{H}_{s}\right]$ of subsystems. 
In quantum mechanics, Heisenberg uncertainty relation imposes Mandelstam-Tamm constraint [39] on the minimum time necessary for the transition of a quantum state $\rho_{1}$ to another perfectly distinguishable state $\rho_{2}$ [23]:

$$
\begin{aligned}
& \Delta t \geqslant \frac{\hbar}{\sqrt{2}} \frac{\cos ^{-1} A\left(\rho_{1}, \rho_{2}\right)}{\left.\sqrt{Q\left(\rho_{1}, \hat{H}\right.}\right)}, \\
& A\left(\rho_{1}, \rho_{2}\right) \equiv \operatorname{tr}\left(\sqrt{\rho_{1}} \sqrt{\rho_{2}}\right), \quad Q(\rho, \hat{H}) \equiv \frac{1}{2}\left|\operatorname{tr}\left([\sqrt{\rho}, \hat{H}]^{2}\right)\right|
\end{aligned}
$$

where $\hat{H}$ is the system's Hamiltonian. Consider $\rho_{1}$ as the state of Universe after selecting and separating an observer and a clock and $\rho_{2}$ as an infinitesimal variation of $\rho_{1}$, that is $\rho_{2}=\rho_{1}+d \rho_{1}$. We assume that the clock is chosen, such that, in (9), minimum time is achieved. Subsequently, (9) becomes:

$$
Q\left(\hat{H}, \rho_{1}\right) d t^{2}=\operatorname{tr}\left(\sqrt{d \rho_{1}}{\sqrt{d \rho_{1}}}^{\dagger} \equiv d s^{2}\right.
$$

This equation is similar to a Riemann metric for a system at rest with respect to the chosen coordinates frame for the parameter space $(r, \theta, \phi, t)$. On the other hand, the r.h.s. of (10) only depends on the variation of state and is independent of the chosen frame for parameters. Therefore, $d s$ is similar to an infinitesimal separation. A coordinate transformation, i.e., $(r, \theta, \phi, t) \rightarrow\left(r^{\prime}, \theta^{\prime}, \phi^{\prime}, t^{\prime}\right)$ does not change state of the Universe and is equivalent to a basis transformation in the Hilbert space. On the other hand, the 1.h.s. of (10) changes. Considering the similarity of (10) to metric equation, we can write $d s^{2}$ as:

$$
g_{00} d t^{\prime 2} \pm g_{i i} d x^{\prime i} d x^{\prime i}=d s^{2}
$$

where we have used Cartesian coordinates in place of spherical. We have chosen parameter transformation such that $g_{0 i}=g_{i 0}=0$. We have also assumed $g_{i j}>0$ and factorized the sign of spatial part of the metric. In these new coordinates, the Hamiltonian associated to the new clock $t^{\prime}$ is $\hat{H}^{\prime}$ and Mandelstam-Tamm relation imposes:

$$
Q^{\prime}\left(\hat{H}^{\prime}, \rho_{1}\right) d t^{\prime 2} \equiv g_{00} d t^{\prime 2} \geqslant \operatorname{tr}\left(\sqrt{d \rho_{1}} \sqrt{d \rho_{1}}{ }^{\dagger}\right)=d s^{2}
$$

For $d s^{2} \geqslant 0$, constraint (12) is only satisfied if the sign of spatial part in (11) and thereby the signature of the metric is negative. We remind that Mandelstam-Tamm constraint does not apply to states that do not fulfill distinguishability condition. In these cases, $d s^{2}<0$ is allowed. In classical view of spacetime, they correspond to spacelike events, where two events/states are not causally related. In quantum mechanics, this can be related to nonlocality [40] and the absence of strict causality. Additionally, the explicit dependence of separation on the density matrix in (10) and (12) and its independence of the coordinate frame of the parameters/spacetime confirms and completes the discussion of Section 4.4 regarding the curved geometry of parameter space and diffeomorphism invariance of subdivided Universe. 


\subsection{Lagrangian of Subsystems}

Finally, the Lagrangian of the Universe after the division to subsystems and selection of reference observer and clock takes the following form:

$$
\begin{aligned}
\mathcal{L}_{U_{s}}= & \int d^{4} x \sqrt{-g}\left[\frac{1}{16 \pi G_{N} \hbar} \sum_{l, m, l^{\prime}, m^{\prime}} \operatorname{tr}\left(L_{l m}^{*}(x) L_{l^{\prime} m^{\prime}}(x) \hat{L}_{l m} \hat{L}_{l^{\prime} m^{\prime}}\right)+\right. \\
& \frac{1}{8\left(\pi G_{N} \hbar\right)^{1 / 2}}\left(\sum_{l, m, a} \operatorname{tr}\left(L_{l m}(x) T_{a}(x) \hat{L}_{l m} \otimes \hat{T}_{a}\right)+\sum_{l m} L_{l m} \operatorname{tr}\left(\hat{L}_{l m} \otimes \mathbb{1}_{G} \rho(x)\right)\right)+ \\
& \left.\frac{1}{4} \sum_{a, b} \operatorname{tr}\left(T_{a}^{*}(x) T_{b}(x) \hat{T}_{a} \hat{T}_{b}\right)+\frac{1}{2} \sum_{a} T_{a} \operatorname{tr}\left(\mathbb{1}_{S U(\infty)} \otimes \hat{T}_{a} \rho(x)\right)\right] .
\end{aligned}
$$

The terms of this Lagrangian can be interpreted as the following. The first term is the Lagrangian for an ensemble of $S U(\infty)$ symmetries of all subsystems, except observer and clock. Amplitudes $L_{l^{\prime} m^{\prime}}(x)$ depend on full $S U(\infty)$ parameter space, which is $(r, \theta, \phi, t)$. Due to the nonlocal algebra (7), we expect that $L_{l^{\prime} m^{\prime}}(x)^{\prime}$ s include derivative terms. Additionally, $L_{l m}$ 's are normalized such that the usual gravitational coupling be explicit. We notice that, if $\hbar G_{N} \propto \hbar^{2} / M_{P}^{2} \rightarrow 0$, the first and the third terms will be canceled. Therefore, the naive classical limit of the model does not include these gravity related terms.

The second term presents gravitational interaction of internal gauge fields and matter, respectively. The third and forth terms together correspond to the Lagrangian of pure gauge fields for local $G$ symmetry and its interaction with matter field. They take the standard form of Yang-Mills models if $T_{a}(x)$ fields are two-forms in the $(3+1) \mathrm{D}$ parameter space.

We leave explicit description of $L_{l^{\prime} m^{\prime}}(x)^{\prime}$ s and $T_{a}$ 's as functionals of spacetime, and determination of semi-classical limit of the Lagrangian for future works. Nonetheless, the Lagrangian (13) is not completely abstract. $L_{l m}$ operators can be expressed as a tensor product of Pauli matrices and regrouped by $r$ and $t$ indices, which have no other role than associating a group of matrices to subsystems. This is because the tensor product of $S U(\infty)$ is homomorphic to itself. However, such expansion is not very useful and practical for analytical calculations, in particular for finding semi-classical limit of the model.

\section{Comparison with Other Quantum Gravity Models}

It is useful to compare this model with string theory and Loop Quantum Gravity (LQG) — the two most popular quantum gravity candidates.

A common aspect of string/superstring theories with the present model is the presence of a 2D manifold in their foundation. However, in contrast to string theories, in which a 2D world sheet is introduced as an axiom without any observational support, the presence of a 2D manifold here is a consequence of the infinite symmetry of the Universe, which has compelling observational support. Moreover, the 2D nature of the underlying Universe manifests itself only when the Universe is considered as a whole. Otherwise, it is always perceived as a $(3+1) \mathrm{D}$ continuum (plus parameters of internal symmetry of subsystems).

In string theory, matter and spacetime are fields living on the 2D world sheet, or equivalently the world sheet can be viewed as being embedded in a multi-dimensional, partially compactified space without any explanation for the origin of such non-trivial structures. On the other hand, in the present model the approach to matter is rather bottom-up. The Cartan decomposition of $S U(\infty)$ to smaller groups, in particular $S U(2)$ means that they can be easily break and separate from the pool of the $S U(\infty)$ symmetry-for instance by quantum correlation between pairs of subsystems-without affecting the infinite symmetry. And indeed it seems to be the case because $S U(2)$ and $S U(3) \subset S U(2) \times S U(2) \cong$ $S U(4)$ are Standard Model symmetries. Additionally, string theory is fundamentally first quantized and string based field theories are considered to be low energy effective descriptions. However, 
as explained in the previous sections, in the present model owing to its infinite dimensional symmetry, Hilbert and Fock spaces are homomorphic and the model can be straightforwardly considered as first or second quantized.

The importance of $S U(2)$ symmetry in the construction of LQG and its presentation as spin foam [20] is shared with the present model. However, $S U(2) \cong S O(3)$ manifold on which Ashtekar variables are defined has its origin in the ADM $(3+1) \mathrm{D}$ formalism, based on the presumption that spacetime and thereby quantum gravity should be formulated in the physical spacetime. Moreover, LQG does not address the origin of matter as the source of gravity or the origin of the Standard Model symmetries. The present model explains both the dimension of spacetime and relation between quantum gravity, matter, and SM symmetries.

A concept that string theory and LQG does not consider-at least not in their foundation-is the fact that in quantum mechanics discrimination between observer and observered is essential, and models which do not consider this concept in their construction-especially when the models is intended to be applied to the whole Universe-are somehow metaphysical, because they implicitly consider that the observer is out of this Universe.

\section{Outline and Future Perspectives}

In this work, we proposed a new approach to quantum gravity by constructing a Universe in which gravity is fundamentally quantic and demonstrated how it may answer some of questions that we raised in the Introduction section regarding gravity and the nature of spacetime. As we have already summarized the model and its results in Section 1.1, here we concentrate on perspectives for further studies.

Understanding nonlocality and differential form of the algebra of subsystem defined by Equation (7) is crucial for finding an algebraic expression for the Lagrangian (13), which, at present, is too abstract. This task is especially important for investigating the semi-classical limit of the model. On the other hand, this Lagrangian describes an open system, because the state of the observer and probably some of degrees of freedom of the clock are traced out. Formulation of the subdivided Universe as an open system should help application of the model to black hole physics and cosmology.

We discussed a bottom-up procedure for the emergence of internal symmetries in Section 4. In particular, we concluded that they should generate stronger couplings between particles/subsystems than gravity. However, this argument does not explain how the hierarchy of couplings arises. We conjecture that clustering of subsystems, which leads to the emergence of internal symmetries, also determines their couplings, probably through processes that are analogous to the formation of moiré super-lattice and strong correlation between electrons in 2D materials. The fact that, in this model, both the Universe as a whole and its subsystems have $S U(\infty)$ symmetry, which is represented by diffeomorphism of $2 \mathrm{D}$ surfaces, means that the necessary ingredients for formation of moiré-like structures are readily available.

In the absence of experimental quantum gravity tests, the ability of models to solve theoretical issues has prominent importance. Among topics that must be addressed black holes and puzzles of information loss in semi-classical approaches have high priority. Because the model studied here is inherently quantic, the first task is finding a purely quantic definition for black holes. Naively, a quantum black hole may be defined as a many particle system in a quantum well in real space. However, we know that quantum field theory in curved spacetime background of black holes leads to Hawking radiation and extraction of energy from black hole. Consequently, in the realm of quantum mechanics, black holes are not really contained in a limited region of space. Their potential well is not perfect and their matter content extends to infinity. Thus, this definition should be considered as an initial condition.

Inflation and dark energy are other issues that should be investigated in the context of this model. Notably, it would be interesting to see whether the topological nature of 2D Lagrangian of the whole Universe can have observable consequences, for instance, as a small but nonzero vacuum energy. 
As for inflation, an exponential decoupling and decoherence of particles/subsystems in the early universe may be interpreted as inflation and an extension of spacetime. These possibilities need detailed investigation.

In conclusion, the inhomogeneous Lorentz transformation may be the classical interface of a much deeper and global realm of a quantum Universe.

Funding: This research received no external funding.

Acknowledgments: The author thanks Institut Henry Poincaré for hospitality and bibliographic assistance during accomplishment of this work.

Conflicts of Interest: The author declares no conflict of interest.

\section{Appendix A. A Very Brief Summary of the Best Studied Quantum Gravity Models}

Introduction of quantum mechanical concepts to general relativity was first mentioned by Einstein himself in his famous 1916 paper. The first detailed work on the topic was by Léon Rosenfeld in 1930 [41], in which the action of Einstein-Hilbert model with matter is quantized by replacing classical variables with hermitian operators, see e.g., [42] for the history of early approaches to quantum gravity. This canonical approach and its modern variants based on the quantization of $3+1$ dimensional Hamiltonian description of dynamics, notably Wheeler-DeWitt (WD) formalism [43,44] and quantum geometrodynamics [45] lead to nonrenormalizable models. ${ }^{13}$ See e.g., [46] for review of other issues of these approaches and their current status.

Another model, inspired by the ADM Hamiltonian formulation of general relativity [47], the Dirac Hamiltonian description of quantum mechanics [48], and the WD approach to QGR is Loop Quantum Gravity (LQG), see e.g., $[49,50]$ and references therein. In this approach, triads defined on a patch of the 3D space — what is called Ashtekar variables [19]—-eplace spatial coordinates and are considered as Hermitian operators acting on the Hilbert space of the Universe. Their conjugate operators form a SU(2) Yang-Mills theory and provide a connection-up to an undefined constant called Immirzi parameter-for the quantized 3D space. However, to implement diffeomorphism of general relativity without referring to a fixed background, the physical quantized entities are holonomies-gauge invariant nonlocal fluxes and Wilson loops defined on 2D surfaces and their boundaries, respectively. Similar to the WD formalism, the LQG Hamiltonian is a constraint, and thereby there is no explicit time in the model [49]. Recently, it is shown that a conformal version of the LQG has an explicit time parameter [51]. But, conformal symmetry must be ultimately broken to induce a mass or distance scale in the model. Other issues in the LQG are lack of explicit global Lorentz invariance, absence of any direct connection to matter, and most importantly quantization of space, that violates Lorentz invariance even when the absence of time parameter in the model is neglected.

Regrading the violation of Lorentz invariance, even if discretization is restricted to distances close to the Planck scale, matter interaction propagates it to larger distances [10]. This issue is also present in other background independent approaches to quantum gravity, in which in one way or another the spacetime is discretized. Examples of such models are symplectic quantum geometry [52] and dynamical triangulations, in which space is assumed to consist of a dynamical lattice $[53,54]$. See also [55] for a recent review of these approaches and [56] for some of their issues, in particular a likely absence of a UV fixed point, which is necessary for renormalizabilty of these models. Therefore, the claimed quantization of space volume or in other words emergence of a fundamental length scale in UV limit of these models is still uncertain. Another example is causal sets-a discretization approach with causally ordered structures [57], see e.g., [58] for a review. They probably suffer from the same issue as other discretization models, notably breaking of Lorentz symmetry, see e.g., [59], but also [60]

13 We should emphasize that references given in this appendix are only examples of works on the subjects on which tens or even hundreds of articles can be found in the literature. 
for counter-arguments. We should remind that all quantum gravity models depend on a length (or equivalently mass) scale, namely the Planck length $L_{P}$ (or mass $M_{P}$ ). Dimensionful quantities need a unit, which does not arise from dimensionless or scale invariant quantities. Therefore, discretization is not a replacement for a dimensionful fundamental constant in quantum gravity models.

Another way of quantizing spacetime without discretization is consideration of a noncommutative spacetime [61,62]. This formalism is in fact one of the earliest proposals for a quantum gravity. More recently this approach is studied in conjunction with other QGR models such as string theory [63] and matrix models [64]. An essential issue of this class of models is their inherent nonlocality that leads to mixing of low and high energy scales [65]. On the other hand, this characteristic might be useful for constraining them, and thereby related QGR models [66].

In early 1980's the discovery of both spin-1 and spin-2 fields in 2D conformal quantum field theories embedded in a $D$-dimensional spacetime-called string models-opened a new era and discipline for seeking a reliable quantum model for gravity, and ultimately unifying all fundamental forces in a Great Unified Theory (GUT). Nambu-Goto and Polyakov string theories were studied in 1970's as candidates for describing strong interaction of hadrons. Although with the establishment of Quantum Chromo-Dynamics (QCD) as the true description of strong nuclear force string theories seemed irrelevant, their potential for quantizing spacetime $[67,68]$ gave them a new role in fundamental particle physics. String and superstring theories became and continue to be by far the most extensively studied candidates of quantum gravity and GUT. ${ }^{14}$

Quantized strings/superstring models are finite and meaningful only for special values of spacetime dimension $D$. For these cases, the central charge of Virasoro algebra or its generalization to affine Lie algebra vanishes when the contribution of all fields, including ghosts of the conformal theory on the 2D world-sheet are taken into account. Without this restriction the theory is infested by anomalies, singularities, and misbehaviour. The allowed dimension is $D=26$ for bosonic string theories and $D=10$ for superstrings. The group manifold on which a viable string model can live is restricted as well. For instance, the allowed symmetry in heterotic Polyakov model is $S O(32)$ or $E_{8} \times E_{8}$. Wess-Zumino-Novikov-Witten (WZNW) models with 2D affine Lie algebras provide more variety of symmetries, including coset groups. However, restriction on dimension/rank of symmetry groups remains the same. Therefore, to make contact with real world, which has $3+1$ dimensions, the remaining dimensions must be compactified.

Initially the inevitable compactification of fields in string models was welcomed because it might explain internal global and local (gauge) symmetries of elementary particles, in a similar manner as in Kaluza-Klein unification of gravity and electromagnetism [71,72]. However, intensive investigations of the topic showed that compactification generates a plethora of possible models. Some of these models may be considered more realistic than others based on the criteria of having a low energy limit containing the Standard Model symmetries. But, unobserved massless moduli, which may make the Universe overdense if they acquire a mass at string or even lower scales, strongly constrain many of string models. Therefore, moduli must be stabilized [73,74]. For instance, they should acquire just enough effective mass to make them a good candidate for dark matter [75]. Moreover, in string theories there is no natural inflation candidate satisfying cosmological observations without fine-tuning. Although moduli are considered as potential candidates for inflation [76], small non-Gaussianity of Cosmic Microwave Background (CMB) anisotropies [77] seems to prefer single field inflation [78]. In addition, single field slow roll inflation may be inconsistent [79] with constraints to be imposed on a scalar field interacting with quantum gravity in the framework of swampland extension of string models landscape [80]. Some researchers still believe that a genuinely non-perturbative formulation of superstring theories may solve many of these issues ${ }^{15}$. However, the absence of any evidence of

14 A textbook description and references to original works can be found in textbooks such as $[69,70]$.

15 Non-supersymmetric string models may have no non-perturbative formulation and should be considered as part of a supersymmetric model, see e.g., Chapter 8 of [70]. 
supersymmetry up to $\sim \mathrm{TeV}$ energies at LHC —-where it was expected, such that it could solve Higgs hierarchy problem [81] —is another disappointing result for string models.

Observation of accelerating expansion of the Universe due to a mysterious dark energy with properties very similar to a cosmological constant-presumably a nonzero but very small vacuum energy-seems to be another big obstacle for string theory [82] as the only quantum gravity candidate including both matter and gravity in its construction. The landscape of string vacua has $\gtrsim 10^{200-500}$ minima-depending on how models are counted [83]. But there is no rule to determine which one is more likely and why the observed density of dark energy-if it is the vacuum energy-is $\sim 10^{123}$ fold less than its expected value, namely $M_{P}{ }^{4}$. To tackle and solve some of these issues, extensions and/or reformulations of string theories have led to their variants such as matrix models [84,85], M-theory, F-theory, and more recently swampland [80] and weak gravity conjecture [38,86], and models constructed based on them.

In early 1999 Randall-Sundrum brane models $[87,88]$ and their variants-inspired by D-branes in toroidal compactification of open strings and propagation of graviton closed strings in the bulk of one or two non-compactified warped extra dimensions-generated a great amount of excitement and were subject of intensive investigations. By confining all fields except gravitons on $4 \mathrm{D}$ branes these models are able to lower the fundamental scale of quantum gravity to $\mathrm{TeV}$ energies-presumably the scale of weak interaction - and explain the apparent weakness of gravitational coupling and high value of Planck mass. Thus, a priori brane models solve the problem of coupling hierarchy in Standard Model of particle physics. In addition, an effective small cosmological constant on the visible brane may be achievable [89,90]. However, brane models, in general, have a modified Friedmann equation, which is strongly constrained by observations [91-93]. Moreover, it is shown that the confinement of gauge bosons on the brane(s) violates gauge symmetries, and if gauge fields propagate to the bulk, so do the matter [94,95]. Nonetheless, some methods for their localization on the brane are suggested [96,97]. On the other hand, observation of ultra high energy cosmic rays constrains the scale of quantum gravity and characteristic scale of warped extra-dimension to $>100 \mathrm{TeV}[98,99]$. This constraint is consistent with other theoretical and experimental issues of brane models, specially in the context of black hole physics, that is instability of macroscopic black holes, nonexistence of an asymptotically Minkowski solution [100,101], and observational constraint [102] on the formation of microscopic black holes in colliders at $\mathrm{TeV}$ energies [103].

In the view of these difficulties more drastic ideas have emerged. Some authors suggests UV/IR correspondence of gravity. They propose that at UV scales graviton quantum condensate behaves asymptotically similar to classical gravity $[104,105]$. Other proposals attracting some interest include the emergence of classical gravity and spacetime from thermodynamics and entropy [106,107] or condensation of more fundamental fields [108,109].

More recently, the development of quantum information theory and its close relation with entanglement of quantum systems, their entropy and the puzzle of information loss in Hawking radiation of black holes have promoted models that interpret gravity and spacetime as an emergent effect of entanglement [110-112] and tensor networks [113,114]. These ideas are in one way or another related to holography principle and Ads/CFT equivalence conjecture [115]. In these models spacetime metric and geometry emerge from tensor decomposition of the Hilbert space of the Universe to entangled subspaces. The resulted structures are interpreted as graphs and a symplectic geometry is associated to them. In the continuum limit the space of graphs can be considered as a quantum spacetime. In a somehow different approach in the same category of models the concept of locality specified by subalgebras is used to decompose the Universe. Local observables belong to spacelike subspaces in a given reference frame/basis $[116,117]$. This means that in these models a background spacetime is implicitly postulated without being precise about its origin and nature. In addition to spacetime, subsystems/subalgebras should somehow present matter. But, it is not clear how they are related. Moreover, the problem of the spacetime dimensionality and how it acquires its observed value 
is not discussed. In any case, investigation of these approaches to quantum gravity is still in its infancy and their theoretical and observational consistency are not fully worked out.

\section{Appendix B. Quantum Mechanics Postulates in Symmetry Language}

In this appendix we reformulate axioms of quantum mechanics à la Dirac [118] and von Neumann [119] with symmetry as a foundational concept:

i. A quantum system is defined by its symmetries. Its state is a vector belonging to a projective vector space called state space representing its symmetry group. Observables are associated to self-adjoint operators. The set of independent observables is isomorphic to subspace of commuting elements of the space of self-adjoint (Hermitian) operators acting on the state space and generates the maximal abelian subalgebra of the algebra associated to symmetry group.

ii. The state space of a composite system is homomorphic to the direct product of state spaces of its components. ${ }^{16}$ In the special case of separable components, this homomorphism becomes an isomorphism. Components may be separable-untangled-in some symmetries and inseparable - entangled-in others. The symmetry group of the states of a composite system is a subgroup of direct product of its components.

iii. Evolution of a system is unitary and is ruled by conservation laws imposed by its symmetries and their representation by the state space.

iv. Decomposition coefficients of a state to eigen vectors ${ }^{17}$ of an observable presents the coherence/degeneracy of the system with respect to its environment according to that observable. Projective measurements by definition correspond to complete breaking of coherence/degeneracy. The outcome of such measurements is the eigen value of the eigen state to which the symmetry is broken. This spontaneous decoherence (symmetry breaking) ${ }^{18}$ reduces the state space to the subspace generated by other independent observables, which represent remaining symmetries/degeneracies.

v. A probability independent of measurement details is associated to eigen values of an observable as the outcome of a measurement. It presents the amount of coherence/degeneracy of the state before its breaking by a projective measurement. Physical processes that determine the probability of each outcome are collectively called preparation. ${ }^{19}$

These axioms are very similar to their analogues in the standard quantum mechanics, except that we do not assume an abstract Hilbert space. The Born rule and classification of the state space as a Hilbert space can be demonstrated using axioms (i) and (v), and properties of statistical distributions [21]. We remind that the symmetry represented by the Hilbert space of a quantum system is in addition to the global $U(1)$ symmetry of states, which leaves probability of outcomes in a projective measurement unchanged. When system is divided to subsystems that can be approximately considered as non-interacting, each subsystem acquire its own local $U(1)$ symmetry. Even in presence of interaction between subsystems, a local $U(1)$ symmetry can be considered, as long as the interaction does not change the Hilbert space of subsystems. We notice that axiom (ii) slightly diverges from its analogue in the standard quantum mechanics. It emphasizes on the fact that the symmetry group represented by a composite system can be smaller than the tensor product of those of its components.

16 Notice that this axiom differentiates between possible states of a composite system, which is the direct product of those of subsystems, and what is actually realized, which can be limited to a subspace of the direct product of individual components and have reduced symmetry.

17 More precisely rays because state vectors differing by a constant are equivalent.

18 Ref. [21] explains why decoherence should be considered as a spontaneous symmetry breaking similar to a phase transition.

19 Literature on the foundation of quantum mechanics consider an intermediate step called transition between preparation and measurement. Here we include this step to preparation or measurement operations and do not consider it as a separate physical operation. 
In particular, entanglement may reduce the dimension of Hilbert space and thereby the rank of symmetry group that it represents.

A corollary of these axioms is that without division of the Universe to system(s) and observer(s) the process of measurement is meaningless. In another word, an indivisible universe is trivial and homomorphic to an empty set. In standard quantum mechanics the necessity of the division of the Universe to subsystems arises in the Copenhagen interpretation, which has many issues, see e.g., [120] for a review. In covariant quantum models and ADM canonical quantization of gravity, in which Hamiltonian is always null and naively the Universe seems to be static, relational definitions of time is based on the division of the Universe to subsystems, see e.g., [27]. Therefore, we conclude that division to subsystems is fundamental concept and must be explicitly included in the construction of quantum cosmology models.

\section{Appendix C. State Space Symmetry and Coherence}

The choice of a Hilbert space $\mathcal{H}$ to present possible states of a system is usually based on the symmetries of its classical Lagrangian. Although these symmetries have usually a finite rank-the number of simultaneously measurable observables-the Hilbert space presenting them may be infinite dimensional. For example, translation symmetry in a 3D space is homomorphic to $U(1) \times U(1) \times U(1)$ and has a global $S U(2) \cong S O(3)$ symmetry under rotation of coordinates. They can be presented by 6 parameters/observables. Thus, the rank of the symmetry is finite. Nonetheless, due to the abelian nature of $U(1)$ group, the Hilbert space of position operator $\mathcal{H}_{X}$ is infinite dimensional. More generally, the dimension of the Hilbert space depends on the dimension of the representation of the symmetry group of Lagrangian and its reducibility. The Hilbert space of a multi-particle system can be considered as a reducible representation of the symmetry, even if single particles are in an irreducible representation. In particular, Fock space of a many-particle system can be presented as an infinite dimensional Hilbert space representing symmetries of the Lagrangian in a reducible manner. This property is important for the construction of the quantum Universe model studied here, because it demonstrates that the infinite size of the physical space can be equally interpreted as manifestation of infinite number of particles/subsystems in a composite Universe.

Ensemble of linear operators acting on a Hilbert space $\mathcal{B}[\mathcal{H}]$ represents $S U(N)$ group where $N$ is the dimension of the Hilbert space $\mathcal{H}$ and can be infinite. As discussed in details in [21] configuration space of classical (statistical) systems have $\otimes^{N} U(1)$ symmetry where each $U(1)$ is isomorphic to the continuous range of values that an observable may have. Thus, quantization extends the symmetry of classical configuration space to $\mathcal{B}[\mathcal{H}]=S U(N) x U(1) \cong U(N) \supset \otimes^{N} U(1)$, where here we have also considered the global $U(1)$ symmetry of the Hilbert space.

Application of linear operators can be interpreted as interaction with another system or more generally with the rest of the Universe. The change of state can be also considered as Positive Operator Valued Measurement (POVM). In particular, a projective measurement and decoherence makes the state completely incoherent $\hat{\rho}_{\text {inc }}$ :

$$
\hat{B} \hat{\rho}_{c} \hat{B}^{\dagger} \rightarrow \hat{\rho}_{i n c}=\sum_{i} \rho_{i} \hat{\rho}_{i}, \quad \hat{\rho}_{i} \equiv|i\rangle\langle i|
$$

where $\hat{B} \in \mathcal{B}[\mathcal{H}],|i\rangle$ is an eigen basis for the measured observable, and subscript ${ }_{\text {inc }}$ means incoherent. We remind that the space of simultaneously observable operators corresponds to the Cartan subalgebra of $\mathcal{B}[\mathcal{H}]$. Coefficients $\rho_{i}$ are probability of occurrence of eigen value of $|i\rangle$ as outcome of the measurement. Because $\hat{\rho}_{i n c}$ is diagonal, completely incoherent states $\hat{\rho}_{i n c}$ represent the Cartan subgroup of $\mathcal{B}[\mathcal{H}]$. A maximally coherent state in the above basis is defined as:

$$
\hat{\rho}_{\operatorname{maxc}} \propto \sum_{i, j}|i\rangle\langle j|
$$


This is a pure state, in which all eigen states have the same occurrence probability in a projective measurement. Notice that due to the projectivity of Hilbert space $\hat{\rho}_{\operatorname{maxc}}$ is unique and application of any other member of $\mathcal{B}[\mathcal{H}]$ reduces its coherence, quantified for instance by fidelity or Fubini-Study metric [22]. More generally, action of $\mathcal{B}[\mathcal{H}]$ members changes coherence of any state which is not completely incoherent. For this reason, we call $S U(N)$ symmetry of $\mathcal{B}[\mathcal{H}]$ the coherence symmetry. ${ }^{20}$

It is useful to remind that in particle physics generators of $\mathcal{B}[\mathcal{H}]$ space physically exist and are not abstract operation of an apparatus controlled by an experimenter. In the Standard Model $\mathcal{B}[\mathcal{H}]$ is generated by vector boson gauge fields in fundamental representation of SM symmetry group. They act on the Hilbert space generated by matter fields. If gravity, which is the only known universal interaction follows the same rule, we should be able to define a Hilbert space for matter on which linear operators representing gravity act. In the model studied here we identify these operators with $\hat{L}$ 's defined in Section 2.

Regarding the example of translational and rotational symmetries of the physical space mentioned earlier, despite the fact that the dimension of the Cartan subalgebra of $\mathcal{B}\left[\mathcal{H}_{X}\right] \cong S U(N \rightarrow \infty)$ is infinite, and a priori there must be infinite simultaneously observable quantities in the physical space, in quantum mechanics only one vector observable is associated to $\mathcal{B}\left[\mathcal{H}_{X}\right]$, namely the position of a particle/system. QFTs define field operators at every point of the space and assume that at equal time operators at different positions commute (or in the case of fermions anti-commute). However, in the formulation of QFT models position is a parameter not an operator. These different interpretations of spacetime highlight the ambiguity of its nature in quantum contexts-as described in question 2 in the Introduction section.

\section{Appendix D. $S U(\infty)$ and Its Polynomial Representation}

Special unitary group $S U(N)$ can be considered as $N$-dimensional representation of $S U(2)$. For this reason generators $T_{l m}^{(N)}$ of the associated Lie algebra $s u(N)$ can be expanded as a matrix polynomial of $N$-dimensional generators of $S U(2)$. Indices $(l, m)$ in these generators are the same as in $S U(2)$ representations: $l=0, \cdots, N-1, m=-l \ldots,+l$. Lie bracket of generators $T_{l m}^{(N)}$ is defined as:

$$
\left[\hat{T}_{l m}^{(N)}, \hat{T}_{l^{\prime} m^{\prime}}^{(N)}\right]=f_{l m, l^{\prime} m^{\prime}}^{(N) l^{\prime \prime}} \hat{T}_{l^{\prime \prime} m^{\prime \prime}}^{(N)}
$$

Structure coefficients $f_{l m, l^{\prime} m^{\prime}}^{(N) l^{\prime \prime} m^{\prime \prime}}$ of $s u(N)$ can be written with respect to $3 \mathrm{j}$ and $6 \mathrm{j}$ symbols, see e.g., [29] for their explicit expression. For $N \rightarrow \infty$, after rescaling these generators $\hat{T}_{l m}^{(N)} \rightarrow(N / i)^{1 / 2} \hat{T}_{l m}^{(N)}$, they satisfy the following Lie brackets:

$$
\left[\hat{L}_{l m}, \hat{L}_{l^{\prime} m^{\prime}}\right]=f_{l m, l^{\prime} m^{\prime}}^{l^{\prime \prime} m^{\prime \prime}} \hat{L}_{l^{\prime \prime} m^{\prime \prime}}
$$

where $\hat{L}_{l m} \equiv \hat{T}_{l m}^{(N \rightarrow \infty)}$ and coefficients $f_{l m, l^{\prime} m^{\prime}}^{l^{\prime \prime} m^{\prime \prime}}$ are $N \rightarrow \infty$ limit of $f_{l m, l^{\prime} m^{\prime}}^{(N) l^{\prime \prime}}$. In addition, it is shown [29] that $\hat{L}_{l m}$ can be expanded with respect to spherical harmonic functions $Y_{l m}(\theta, \phi)$ defined on a sphere, i.e., the manifold associated to $S U(2)$ :

$$
\begin{aligned}
& \hat{L}_{l m}=\frac{\partial Y_{l m}}{\partial \cos \theta} \frac{\partial}{\partial \phi}-\frac{\partial Y_{l m}}{\partial \phi} \frac{\partial}{\partial \cos \theta} \\
& \hat{L}_{l m} Y_{l^{\prime} m^{\prime}}=-\left\{Y_{l m}, Y_{l^{\prime} m^{\prime}}\right\}=-f_{l m, l^{\prime} m^{\prime}}^{l^{\prime \prime} m^{\prime \prime}} Y_{l^{\prime \prime} m^{\prime \prime}} \\
& \{\mathrm{f}, \mathrm{g}\} \equiv \frac{\partial \mathrm{f}}{\partial \cos \theta} \frac{\partial \mathrm{g}}{\partial \phi}-\frac{\partial \mathrm{f}}{\partial \phi} \frac{\partial \mathrm{g}}{\partial \cos \theta}, \quad \forall \mathrm{f}, \mathrm{g} \text { defined on the sphere }
\end{aligned}
$$

20 In some quantum information literature coherence symmetry is called asymmetry [23]. In this work we call it coherence symmetry or simply coherence to remind that its origin is quantum degeneracy and indistinguishability/symmetry of states before a projective observation. 
where $\theta=[0, \pi]$ and $\phi=[0,2 \pi]$ are angular coordinates and $\{f, g\}$ is the Poisson bracket of continuous functions $f$ and $g$ on the sphere. We notice that although generators $\hat{L}_{l m}$ are linear combination of $\partial / \partial(\cos \theta)$ and $\partial / \partial \phi$, the latter operators cannot be considered as generators of $S U(N \rightarrow \infty)$, because they commute with each other and generate only the abelian subspace of $S U(\infty)$ group.

Using (A5)-(A7), coefficients $f_{l m, l^{\prime} m^{\prime}}^{l^{\prime \prime} m^{\prime \prime}}$ can be determined:

$$
f_{l m, l^{\prime} m^{\prime}}^{l^{\prime \prime} m^{\prime \prime}}=\frac{\left(2 l^{\prime \prime}+1\right)}{4 \pi} \int d^{2} \Omega Y_{l^{\prime \prime} m^{\prime \prime}}^{*}\left\{Y_{l m}, Y_{l^{\prime} m^{\prime}}\right\}, \quad Y_{l m}^{*}=Y_{l,-m} \quad d^{2} \Omega \equiv d(\cos \theta) d \phi
$$

Here we normalize $Y_{l m}$ such that:

$$
\int d^{2} \Omega Y_{l^{\prime} m^{\prime}}^{*} Y_{l m}=\frac{4 \pi}{(2 l+1)} \delta_{l l^{\prime}} \delta_{m m^{\prime}}
$$

Although $\hat{L}_{l m}$ is defined in discrete $(l, m)$ space—analogous to a discrete Fourier mode-we can use inverse expansion to define operators which depend only on continuous angular coordinates:

$$
\hat{L}(\theta, \phi) \equiv \sum_{l, m} Y_{l m}^{*} \hat{L}_{l m}
$$

As $\{\hat{L}(\theta, \phi)\}$ are linear in $\hat{L}_{l m}$ and contain all these generators, they are also generators of $S U(N \rightarrow$ $\infty) \cong \operatorname{Diff}\left(S_{2}\right)$ and coefficients in their Lie bracket is expressed with respect to $\theta$ and $\phi$ as:

$$
\mathrm{f}\left((\theta, \phi),\left(\theta^{\prime}, \phi^{\prime}\right) ;\left(\theta^{\prime \prime}, \phi^{\prime \prime}\right)\right)=\sum_{l m, l^{\prime} m^{\prime}, l^{\prime \prime} m^{\prime \prime}} Y_{l m}^{*}(\theta, \phi) Y_{l^{\prime} m^{\prime}}^{*}\left(\theta^{\prime}, \phi^{\prime}\right) Y_{l^{\prime \prime} m^{\prime \prime}}\left(\theta^{\prime \prime}, \phi^{\prime \prime}\right) f_{l m, l^{\prime} m^{\prime}}^{l^{\prime \prime} m^{\prime \prime}}
$$

Coefficients $f$ are anti-symmetric with respect to the first two sets of parameters and can be considered as a 2 -form on the sphere, and Lie algebra of $\hat{L}(\theta, \phi)$ operators as:

$$
\left[\hat{L}\left(\theta_{1}, \phi_{1}\right), \hat{L}\left(\theta_{2}, \phi_{2}\right)\right]=\int d \Omega_{3} f\left(\left(\theta_{1}, \phi_{1}\right),\left(\theta_{2}, \phi_{2}\right) ;\left(\theta_{3}, \phi_{3}\right)\right) \hat{L}\left(\theta_{3}, \phi_{3}\right)
$$

Operators $\hat{L}(\theta, \phi)$ are continuous limits of $\hat{L}_{l m}$ 's and both set of generators are vectors and live on the tangent space of the sphere.

\section{Appendix E. Cartan Decomposition of $S U(\infty)$}

Representations of $s u(N)$ algebra can be decomposed to direct sum of smaller $s u$ algebras, see e.g., [34] and references therein. In the case of $S U(\infty)$ the fact that its algebra is homomorphic to Poisson brackets of spherical harmonic functions, which in turn correspond to representations of $S U(2) \cong S O(3)$, means that $s u(\infty)$ algebra should be expandable as direct sum of representations of $S U(2)$, see e.g., $[29,30]$ for the proof. Thus, up to a normalization factor depending only on $l$, generators of $s u(\infty)$ algebra $\hat{L}_{l m}$ can be expanded as:

$$
\hat{L}_{l m}=\mathcal{R} \sum_{i_{\alpha}=1,2,3, \alpha=1, \cdots, l} a_{i_{1}, \cdots i_{l}}^{(m)} \sigma_{i_{1}} \cdots \sigma_{i_{l}}
$$

where $\sigma_{i_{\alpha}}{ }^{\prime}$ s are $N \rightarrow \infty$ representation of Pauli matrices [29]. Coefficients $a^{(m)}$ are determined from expansion of spherical harmonic functions with respect to spherical description of Cartesian coordinates, see [29] for details. This explicit description shows that up to a constant factor $\hat{L}_{l m}$ operators can be considered as tensor product of $2 \times 2$ Pauli matrices, and $S U(\infty) \cong S U(2) \otimes S(2) \otimes \ldots$ This relation can be understood from properties of $S U(N)$ group. Specifically, $S U(N) \supseteq S U(N-K) \otimes$ $S U(K)$. For $N \rightarrow \infty$ and finite $K, S U(N-K \rightarrow \infty) \cong S U(\infty)$. Therefore $S U(\infty)$ is homomorphic to infinite tensor product of $S U$ groups of finite rank, in particular $S U(2)$-the smallest non-abelian $S U$ group. This shows that $S U(2)$ group, which has a key role in some quantum gravity models, 
notably in LQG, simply presents a mathematical description rather than a fundamental physical entity. The description of $S U(\infty)$ as tensor product of $S U(2)$ is comparable with Fourier transform, which presents the simplest decomposition to orthogonal functions, but can be replaced by another orthogonal function. It is only the application that determines which one is more suitable.

Appendix E.1. Eigen Functions of $\hat{L}(\theta, \phi)$ and $\hat{L}_{l m}$

We define eigen functions of $\hat{L}(\theta, \phi)$ and $\hat{L}_{l m}$ operators as the followings:

$$
\begin{aligned}
\hat{L}(\theta, \phi) \eta(\theta, \phi) & =N \eta(\theta, \phi) \\
\hat{L}_{l m} \zeta_{l m} & =N^{\prime} \zeta_{l m}
\end{aligned}
$$

where $N$ and $N^{\prime}$ are constants ${ }^{21}$, but $N^{\prime}$ may depend on $(l, m)$. Using definition of $\hat{L}(\theta, \phi)$ and $\hat{L}_{l m}$ and properties of spherical harmonic functions, solutions of Equations (A14) and (A15) are obtained as:

$$
\begin{gathered}
\left\{\begin{array}{l}
\eta(\theta, \phi)=i N \sum_{l m} \frac{(l+m) !}{m A_{l}(l-m) !}\left[\mathcal{F}_{l m}(\cos \theta)-\mathcal{F}_{l m}\left(\cos \theta_{0}(t)\right)\right]+\eta\left(\theta_{0}(t)\right) \\
\phi+H(\cos \theta)=-\left[H\left(\cos \theta_{0}(t)\right)-\phi_{0}(t)\right]
\end{array}\right. \\
A_{l} \equiv \sqrt{\frac{4 \pi}{2 l+1}} \quad \mathcal{F}_{l m} \equiv \int d(\cos \theta)\left|P_{l m}(\cos \theta)\right|^{-2} \\
H(\cos \theta) \equiv \int d(\cos \theta) \frac{\sum_{l m} \frac{A_{l}(l-m) !}{(l+m) !} \frac{\left.\partial P_{l m} \cos \theta\right|^{2}}{2 \partial \cos \theta}}{\sum_{l^{\prime} m^{\prime}} \frac{i m^{\prime} A_{l^{\prime}}\left(l^{\prime}-m^{\prime}\right) !}{\left(l^{\prime}+m^{\prime}\right) !}\left|P_{l^{\prime} m^{\prime}}(\cos \theta)\right|^{2}}
\end{gathered}
$$

where $t$ parameterizes tangent surface at initial point $\left(\theta_{0}, \phi_{0}\right)$. Elimination of this parameter from two equations in (A16) determines $\eta(\theta, \phi)$ for a set of initial conditions. Because the second equation does not depend on $N$, without loss of generality we can scale initial value $\eta\left(\theta_{0}\right) \rightarrow i N \eta\left(\theta_{0}\right)$. With this choice the eigen value $N$ can be factorized, and because Hilbert space is projective, $N$ can be considered as an overall normalization factor and irrelevant for physics. Therefore, each set of parameters $(\theta, \phi)$ present a unique pointer state for the Hilbert space.

In the same way we can calculate eigen functions of $\hat{L}_{l m}$ as a parametric function:

$$
\begin{array}{r}
\left\{\begin{array}{r}
\zeta_{l m}(\theta)=-N^{\prime} e^{m^{2} W_{l m}\left(\theta_{0}\right)} \sqrt{\frac{(l+m) !}{(l-m) !}}\left[Z_{l m}(\theta)-Z_{l m}\left(\theta_{0}(t)\right)\right]+\zeta_{l m}\left(\theta_{0}(t)\right) \\
\phi-i m W_{l m}(\theta)=\phi_{0}(t)-i m W_{l m}\left(\theta_{0}(t)\right)
\end{array}\right. \\
W_{l m} \equiv \int d(\cos \theta) \frac{\left(1-\cos \theta^{2}\right) P_{l m}(\cos \theta)}{(l-m+1) P_{(l+1) m}(\cos \theta)}-(l+1) P_{l m}(\cos \theta) \\
Z_{l m} \equiv \int d(\cos \theta) \frac{e^{-m^{2}} W_{l m}(\cos \theta)}{(l-m+1) P_{(l+1) m}(\cos \theta)}-(l+1) P_{l m}(\cos \theta)
\end{array}
$$

Similar to $\eta(\theta, \phi)$, redefinition of initial value $Z_{l m}\left(\theta_{0}(t)\right) \rightarrow Z_{l m}\left(\theta_{0}(t)\right) N^{\prime} e^{m^{2} W_{l m}\left(\theta_{0}\right)}$ leads to a unique eigen function for $\hat{L}_{l m}$.

21 A priori $N$ and $N^{\prime}$ can depend on $(\theta, \phi)$. However, their dependence on angular parameters can be included in $\eta$. Therefore, only constant eigen values matter. 
Considering diffeomorphism invariance of the model, it is always possible to redefine coordinates such that $\theta=$ const. and $\phi=$ const. constitute a basis and any state can be written as:

$$
|\psi\rangle=\int d^{2} \Omega \psi(\theta, \phi)|\theta, \phi\rangle
$$

Thus, as explained in the main text, vectors of the Hilbert space representing $S U(\infty)$ are complex functions on $2 \mathrm{D}$ surfaces. As $S U(\infty) \cong \operatorname{Diff}\left(S_{2}\right)$, the range of $(\theta, \phi)$ is $\theta=[0 ., \pi]$ and $\phi=[0 ., 2 \pi)$. However, $S U(\infty)$ may be represented by diffeo-surfaces of higher genus. In this case $\left|\theta+n \pi, \phi+2 n^{\prime} \pi\right\rangle$ for any integer $n$ and $n^{\prime}$ may present different states. States can be also expanded with respect to $|l, m\rangle$ [29].

\section{Appendix E.2. Dynamics Equations of the Universe before its Division to Subsystems}

The equilibrium solution for Lagrangian $\mathcal{L}_{U}$ in (3) can be determined by variation with respect to $L_{l m}$ and components of the state $\rho$ in an orthogonal basis of the Hilbert space. In absence of environment for the whole Universe, $\rho$ is pure and can be written as $\rho=|\psi\rangle\langle\psi|$, where $\psi\rangle$ is an arbitrary vector in the Hilbert space $\mathcal{H}_{U}$. As discussed in Section 2 and explicitly shown in Appendix E.1, vectors of $\mathcal{H}_{U}$ correspond to complex functions of angular coordinates $(\theta, \phi)$ of the diffeo-surface, and can be expanded with respect to spherical harmonic functions. Nonetheless, here we follow the usual bracket notation of quantum mechanics and call states of this orthogonal basis $|l, m\rangle$, where $l \in \mathbb{Z} / 2,-l \leqslant m \leqslant$ $+l$. In this basis $|\psi\rangle=\sum_{l, m} \psi_{l m}|l, m\rangle$ and $\rho=\sum_{l, m, l^{\prime}, m^{\prime}} \psi_{l, m} \psi_{l^{\prime}, m^{\prime}}^{*}|l, m\rangle\left\langle l^{\prime}, m^{\prime}\right|$. After this decomposition dynamics equations are expressed as:

$$
\begin{aligned}
& \frac{\partial \mathcal{L}_{U}}{\partial \psi_{l m}}=\sum_{l^{\prime}, m^{\prime}, l^{\prime \prime}, m^{\prime \prime}} L_{l^{\prime \prime} m^{\prime \prime}} \psi_{l^{\prime} m^{\prime}}^{*}\left\langle l^{\prime} m^{\prime}\left|\hat{L}_{l^{\prime \prime} m^{\prime \prime}}\right| l, m\right\rangle \\
& \frac{\partial \mathcal{L}_{U}}{\partial L_{l m}}=\sum_{l^{\prime}, m^{\prime}, l^{\prime \prime}, m^{\prime \prime}} \psi_{l^{\prime \prime} m^{\prime \prime}}^{*} \psi_{l^{\prime \prime} m^{\prime \prime}}\left\langle l^{\prime}, m^{\prime}\left|\hat{L}_{l m}\right| l^{\prime \prime} m^{\prime \prime}\right\rangle+2 L_{l m} \operatorname{tr}\left(\hat{L}_{l m} \hat{L}_{l m}\right)
\end{aligned}
$$

Because $\hat{L}_{l m}$ is a generator of $S U(\infty)$, the last term in (A24) is a constant depending only on $l$ and normalization of generators. Thus, we define $C_{l} \equiv \operatorname{tr}\left(\hat{L}_{l m} \hat{L}_{l m}\right)$. Using description of $\hat{L}_{l m}$ in (A13) to tensor product of Pauli matrices, we conclude that $\hat{L}_{l m}\left|l^{\prime}, m^{\prime}\right\rangle \neq 0$ only for $l \geqslant l^{\prime}$ and consists of linear combination of $\left|l^{\prime \prime}, m^{\prime \prime}\right\rangle$ states. On the other hand, $\left\langle l^{\prime}, m^{\prime} \mid l, m\right\rangle=\delta_{l l^{\prime}} \delta_{m m^{\prime}}$. Thus, $\left\langle l^{\prime}, m^{\prime}\left|\hat{L}_{l m}\right| l^{\prime \prime} m^{\prime \prime}\right\rangle$ is nonzero only for terms with equal $l$ indices and we can solve (A24) for $L_{l m}$ as the following:

$$
L_{l m}=-\frac{1}{2 C_{l}} \sum_{\left|m^{\prime}\right|,\left|m^{\prime \prime}\right| \leqslant l, m+m^{\prime}+m^{\prime \prime}=0} \psi_{l m^{\prime}}^{*} \psi_{l m^{\prime \prime}}\left\langle l, m^{\prime}\left|\hat{L}_{l m}\right| l m^{\prime \prime}\right\rangle
$$

By applying this solution to (A23) and using properties of $\hat{L}_{l m}$ and $|l, m\rangle$ we find:

$$
\begin{aligned}
& \sum_{m^{\prime \prime}, m^{\prime \prime}} \psi_{l,-\left(m+m^{\prime}\right)}^{*} \psi_{l,-\left(m+m^{\prime \prime}\right)}^{*} \psi_{l m^{\prime}}\left\langle l,-\left(m+m^{\prime \prime}\right)\left|\hat{L}_{l m^{\prime \prime}}\right| l, m\right\rangle\left\langle l,-\left(m^{\prime}+m^{\prime \prime}\right)\left|\hat{L}_{l m^{\prime \prime}}\right| l, m^{\prime}\right\rangle=0 . \\
& \quad\left|m^{\prime}\right|,\left|m^{\prime}\right|,\left|m+m^{\prime \prime}\right|,\left|m^{\prime}+m^{\prime \prime}\right|, \leqslant l, \forall l \in \mathbb{Z} / 2
\end{aligned}
$$

Considering independence and orthogonality of $|l, m\rangle$ states, this equation is satisfied only if $\psi_{l m}=0,|m| \leqslant l, \forall l$. Thus, equilibrium solution of the Lagrangian $\mathcal{L}_{U}$ is a trivial vacuum.

\section{References}

1. Eppley, K.; Hanna, E. The Necessity of Quantizing the Gravitational Field. Found. Phys. 1977, 7, 51. [CrossRef]

2. Ziaeepour, H. And what if gravity is intrinsically quantic? J. Phys. Conf. Ser. 2009, 174, 012027 arXiv:0901.4634. 
3. Barrow, J.D.; Magueijo, J. A contextual Planck parameter and the classical limit in quantum cosmology. arXiv 2020, arXiv:2006.16036.

4. Kolb, E.W.; Turner, M.S. The Early Universe; Addison-Wesley Publishing Company: Boston, MA, USA, 1991.

5. Utiyama, R. Invariant theoretical interpretation of interaction. Phys. Rev. 1956, 101, 1597. [CrossRef]

6. Kibble, T.W.B. Lorentz invariance and the gravitational field. J. Math. Phys. 1961, 2, 212. [CrossRef]

7. Coleman, S.; Mandula, J. All possible symmetries of the $S$ matrix. Phys. Rev. 1967, 159, 1251. [CrossRef]

8. Haag, R.; Lopuszanski, J.T.; Sohnius, M. All possible generators of supersymmetries of the $S$ matrix. Nucl. Phys. B 1975, 88, 257. [CrossRef]

9. Percacci, R. Mixing internal and spacetime transformations: Some examples and counterexamples. J. Phys. A 2008, 41, 335403. [CrossRef]

10. Collins, J.; Perez, A.; Sudarsky, D.; Urrutia, L.; Vucetich, H. Lorentz invariance and quantum gravity: An additional fine-tuning problem? Phys. Rev. Lett. 2004, 93, 191301. [CrossRef]

11. Asenbaum, P.; Overstreet, C.; Kim, M.; Curti, J.; Kasevich, M.A. Atom-Interferometric test of the equivalence principle at the $10^{12}$ level. arXiv 2020, arXiv:2005.11624.

12. Abdo, A.A.; Ackermann, M.; Ajello, M.; Asano, K.; Atwood, W.B.; Axelsson, M.; Baldini, L.; Ballet, J.; Barbiellini, G.; Baring, M.G.; et al. A limit on the variation of the speed of light arising from quantum gravity effects. Nature 2009, 462, 331. [CrossRef] [PubMed]

13. Wilczek, F. Riemann-Einstein Structure from Volume and Gauge Symmetry. Phys. Rev. Lett. 1998, 80, 4851. [CrossRef]

14. Torres-Gomez, A.; Krasnov, K. Gravity-Yang-Mills-Higgs unification by enlarging the gauge group. Phys. Rev. D 2010, 81, 085003. [CrossRef]

15. Barrett, J.W.; Kerr, S. Gauge gravity and discrete quantum models. arXiv 2013, arXiv:1309.1660.

16. Birrell, N.D.; Davies, P.C.W. Quantum Fields in Curved Space; Cambridge University Press: Cambridge, UK, 1982.

17. Parker, L.; Toms, D. Quantum Field Theory in Curved Spacetime; Cambridge University Press: Cambridge, UK, 2009.

18. Ziaeepour, H. Issues with vacuum energy as the origin of dark energy. Mod. Phys. Lett. A 2012, $27,1250154$. [CrossRef]

19. Ashtekar, A. New Variables for Classical and Quantum Gravity. Phys. Rev. Lett. 1986, 57, 2244. [CrossRef]

20. Livine, E.R. Projected Spin Networks for Lorentz connection: Linking Spin Foams and Loop Gravity. Class. Quant. Grav. 2002, 19, 5525. [CrossRef]

21. Ziaeepour, H. Symmetry as a foundational concept in Quantum Mechanics. J. Phys. Conf. Ser. 2015, 626, 012074. [CrossRef]

22. Baumgratz, T.; Cramer, M.; Plenio, M.B. Quantifying Coherence. Phys. Rev. Lett. 2014, 113, 140401. [CrossRef]

23. Mondal, D.; Datta, C.; Sazim, S. Quantum Coherence Sets The Quantum Speed Limit For Mixed States. Phys. Lett. A 2016, 380, 689. [CrossRef]

24. Zanardi, P.; Lidar, D.; Lloyd, S. Quantum tensor product structures are observable-induced. Phys. Rev. Lett. 2004, 92, 060402. [CrossRef]

25. Mazenc, E.A.; Ranard, D. Target Space Entanglement Entropy. arXiv 2019, arXiv:1910.07449.

26. Page, D.N.; Wootters, W.K. Evolution without evolution: Dynamics described by stationary observables. Phys. Rev. D 1983, 27, 2885. [CrossRef]

27. Hoehn, P.A.; Smith, A.R.H.; Lock, M.P.E. The Trinity of Relational Quantum Dynamics. arXiv 2019, arXiv:1912.00033.

28. Cao, C.; Carroll, S.M.; Michalakis, S. Space from Hilbert Space: Recovering Geometry from Bulk Entanglement. Phys. Rev. D 2017, 95, 024031. [CrossRef]

29. Hoppe, J. Quantum Theory of a Massless Relativistic Surface and a Two-dimensional Bound State Problem. Ph.D. Thesis, MIT, Cambridge, MA, USA, 1982.

30. Floratos, E.G.; Iliopoulos, J.; Tiktopoulos, G. A note on $S U(\infty)$ classical Yang-Mills theories. Phys. Lett. B 1989, 217, 285. [CrossRef]

31. Hoppe, J. Diffeomorphism, Group, Quantization, and SU( $\infty)$. Int. J. Mod. Phys. A 1989, 4, 5235. [CrossRef]

32. Hoppe, J.; Schaller, P. Infinitely Many Versions of $S U(\infty)$. Phys. Lett. B 1990, 237, 407. [CrossRef]

33. Zunger, Y. Why Matrix theory works for oddly shaped membranes. Phys. Rev. D 2001, 64 , 086003. [CrossRef] 
34. Su, Z-Y. A Scheme of Cartan Decomposition for su(N). arXiv 2006, arXiv:quant-ph/0603190.

35. Jacobson, T. Thermodynamics of Spacetime: The Einstein Equation of State. Phys. Rev. Lett. 1995, 75, 1260. [CrossRef]

36. Kitaev, A. Anyons in an exactly solved model and beyond. Ann. Phys. 2006, 321, 2. [CrossRef]

37. Nakamura, J.; Liang, S.; Gardner, G.C.; Manfra, M.J. Direct observation of anyonic braiding statistics at the $v=1 / 3$ fractional quantum Hall state. arXiv 2006, arXiv:2006.14115.

38. Arkani-Hamed, N.; Motl, L.; Nicolis, A.; Vafa, C. The String Landscape, Black Holes and Gravity as the Weakest Force. J. High Energy Phys. 2007, 06, 060. [CrossRef]

39. Mandelstam, L.; Tamm, I. The Uncertainty Relation Between Energy and Time in Non-relativistic Quantum Mechanics. J. Phys. (USSR) 1945, 9, 249.

40. Oppenheim, J.; Wehner, S. The Uncertainty Principle Determines Nonlocality of Quantum Mechanics. Science 2010, 330, 1072. [CrossRef]

41. Rosenfeld, L. Zur Quantelung der Wellenfelder. Annal der Physik 1930, 397, 113. [CrossRef]

42. Rocci, A. On first attempts to reconcile quantum principles with gravity. J. Phys. Conf. Ser. 2013, 470, 012004. [CrossRef]

43. Dewitt, B. Quantum Theory of Gravity. I. The Canonical Theory. Phys. Rev. 1967, 160, 1113. [CrossRef]

44. Hartle, J.B.; Hawking, S.W. Wave function of the Universe. Phys. Rev. D 1983, 28, 2960. [CrossRef]

45. Wheeler, J.A. On the nature of quantum geometrodynamics. Ann. Phys. 1957, 2, 604. [CrossRef]

46. Kiefer, C. Quantum geometrodynamics: Whence, whither? Gen. Rel. Grav 2009, 41, 877. [CrossRef]

47. Arnowitt, R.; Deser, S.; Misner, C. Dynamical Structure and Definition of Energy in General Relativity. Phys. Rev. 1959, 116, 1322. [CrossRef]

48. Dirac, P. Generalized Hamiltonian dynamics. Proc. Roy. Soc. Lond. A 1958, 246, 326. [CrossRef]

49. Rovelli, C. Quantum Gravity; Cambridge University Press: Cambridge, UK, 2004.

50. Ashtekar, A.; Lewandowski, J. Background Independent Quantum Gravity: A Status Report. Class. Quant. Grav. 2004, 21, R53. [CrossRef]

51. Wang, C.H.; Rodrigues, D.P.F. Closing the gaps in quantum space and time: Conformally augmented gauge structure of gravitation. Phys. Rev. D 2018, 98, 124041. [CrossRef]

52. Crnkovic, C. Symplectic geometry of covariant phase space. Class. Quant. Grav. 1988, 5, 1557. [CrossRef]

53. Migdal, A.A. Quantum Gravity as Dynamical Triangulation. In Two Dimensional Quantum Gravity and Random Surfaces; Gross, D.J., Piran, T., Weinberg, S., Eds.; World Scientific: Singapore, 1991; pp. 41-79.

54. Ambjorn, J.; Carfora, M.; Marzuoli, A. The Geometry of Dynamical Triangulations; Springer: Berlin/Heidelberg, Germany, 1997.

55. Ambjørn, J.; Görlich, A.; Jurkiewicz, J.; Loll, R. Quantum Gravity via Causal Dynamical Triangulations. In Springer Handbook of Spacetime; Ashtekar, A., Petkov, V., Eds.; Springer Handbooks; Springer: Berlin/Heidelberg, Germany, 2014.

56. Ambjorn, J.; Gizbert-Studnicki, J.; Goerlich, A.; Jurkiewicz, J.; Loll, R. Renormalization in quantum theories of geometry. arXiv 2002, arXiv:2002.01693.

57. Sorkin, R.D. A Unitary Substitute for Continuous Topology. Int. J. Theor. Phys. 1991, 30, 923. [CrossRef]

58. Dowker, F. Causal sets and the deep structure of spacetime. In 100 Years of Relativity: Space-Time Structure-Einstein and Beyond; Ashtekar, A., Ed.; World Scientific: Singapore, 2005; p. 445.

59. Mattingly, D. Causal sets and conservation laws in tests of Lorentz symmetry. Phys. Rev. D 2008, 77, 125021. [CrossRef]

60. Dowker, F.; Sorkin, R.D. Symmetry-breaking and zero-one law. Class. Quant. Grav. 2020, 37, 15. [CrossRef]

61. Snyder, H.S. Quantized Space-Time. Phys. Rev. 1947, 71, 38. [CrossRef]

62. Yang, C.N. On quantized space-time. Phys. Rev. 1947, 72, 874. [CrossRef]

63. Seiberg, N.; Witten, E. String Theory and Noncommutative Geometry. J. High Energy Phys. 1999, $9909,032$. [CrossRef]

64. Steinacker, H. Emergent Gravity from Noncommutative Gauge Theory. J. High Energy Phys. 2007, $0712,049$. [CrossRef]

65. Amelino-Camelia, G.; Mandanici, G.; Yoshida, K. On the IR/UV mixing and experimental limits on the parameters of canonical noncommutative spacetimes. J. High Energy Phys. 2004, 0401, 037. [CrossRef]

66. Hogan, C. Quantum Geometry and Interferometry. In Proceedings of the 9th LISA Symposium; Binétruy, P., Plagnol, E., Eds.; Astronomical Society of the Pacific: San Francisco, CA, USA, 2013. 
67. Polyakov, A.M. Quantum geometry of bosonic string. Phys. Lett. B 1981, 103, 207. [CrossRef]

68. Polyakov, A.M. Quantum geometry of fermionic string. Phys. Lett. B 1981, 103, 211. [CrossRef]

69. Green, M.B.; Schwarz, J.H.; Witten, E. Superstring Theory I E II; Cambridge University Press: Cambridge, UK, 1987.

70. Polchinski, J. String Theory I \& II; Cambridge University Press: Cambridge, UK, 2005.

71. , Kaluza, T. Zum Unitätsproblem der Physik. Sitz. Preuss. Akad. Wiss. Phys. Math. 1921, K1, 966.

72. Klein, O. Quantentheori und fünfdimensionale Relativistätstheori. Zeits Phys. 1926, 37, 895. [CrossRef]

73. Nakamura, S.; Yamaguchi, M. Gravitino Production from Heavy Moduli Decay and Cosmological Moduli Problem Revived. Phys. Lett. B 2006, 638, 389. [CrossRef]

74. Davis, S.C.; Postma, M. Successfully combining SUGRA hybrid inflation and moduli stabilisation. J. Cosmol. Astrop. Phys. 2008, 0804, 022.

75. Shih, D. Pseudomoduli Dark Matter. J. High Energy Phys. 2009, 0909, 046. [CrossRef]

76. Dimopoulos, K.; Axenides, M. Hybrid Inflation without Flat Directions and without Primordial Black Holes. J. Cosmol. Astrop. Phys. 2005, 0506, 008. [CrossRef]

77. Planck Collaboration. Planck 2018 results. IX. Constraints on primordial non-Gaussianity. Astron. Astrophys. 2020, 641, A9. [CrossRef]

78. Maldacena, J. Non-Gaussian features of primordial fluctuations in single field inflationary models. J. High Energy Phys. 2003, 0305, 013. [CrossRef]

79. Kinney, W.H.; Vagnozzi, S.; Visinelli, L. The zoo plot meets the swampland: Mutual (in)consistency of single-field inflation, string conjectures, and cosmological data. Class. Quant. Grav. 2019, 36, 117001. [CrossRef]

80. Vafa, C. The String Landscape and the Swampland. arXiv 2005, arXiv:hep-th/0509212.

81. Susskind, L. Dynamics of spontaneous symmetry breaking in the Weinberg-Salam theory. Phys. Rev. D 1979, 20, 2619. [CrossRef]

82. Bousso, R. The Cosmological Constant Problem, Dark Energy, and the Landscape of String Theory. In Proceedings of the Subnuclear Physics: Past, Present and Future, Vatican City, Vatican, 30 October-2 November 2011; Pontifical Academy of Sciences: Vatican City, Vatican, 2011.

83. Kumar, J. A Review of Distributions on the String Landscape. Int. J. Mod. Phys. A 2006, 21, 3441. [CrossRef]

84. Banks, T.; Fischler, W.; Shenker, S.H.; Susskind, L. M Theory As A Matrix Model: A Conjecture. Phys. Rev. D 1997, 55, 5112. [CrossRef]

85. Dijkgraaf, R.; Verlinde, E.; Verlinde, H. Matrix String Theory. Nucl. Phys. B 1997, 500, 43. [CrossRef]

86. Banks, T.; Johnson, M.; Shomer, A. A note on Gauge Theories Coupled to Gravity. J. High Energy Phys. 2006, 0609, 049. [CrossRef]

87. Randall, L.; Sundrum, R. A Large Mass Hierarchy from a Small Extra Dimension. Phys. Rev. Lett. 1999, 83, 3370. [CrossRef]

88. Randall, L.; Sundrum, R. An Alternative to Compactification. Phys. Rev. Lett. 1999, 83, 4690. [CrossRef]

89. Steinhardt, P.J. General considerations of the cosmological constant and the stabilization of moduli in the brane-world picture. Phys. Lett. B 1999, 462, 41. [CrossRef]

90. Deffayet, C.; Dvali, G.; Gabadadze, G. Accelerated Universe from Gravity Leaking to Extra Dimensions. Phys. Rev. D 2002, 65, 044023. [CrossRef]

91. Franceschini, R.; Giudice, G.F.; Giardino, P.P.; Lodone, P.; Strumia, A. LHC bounds on large extra dimensions. J. High Energy Phys. 2011, 1105, 092. [CrossRef]

92. Alcaniz, J.S.; Zhu, Z-H. Complementary Constraints on Brane Cosmology. Phys. Rev. D 2005, 71, 083513. [CrossRef]

93. Lazkoz, R.; Maartens, R.; Majerotto, E. Observational constraints on phantom-like braneworld cosmologies. Phys. Rev. D 2006, 74, 083510. [CrossRef]

94. Dubovsky, S.L.; Rubakov, V.A.; Tinyakov, P.G. Brane world: Disappearing massive matter. Phys. Rev. D 2000, 62, 105011. [CrossRef]

95. Dubovsky, S.L.; Rubakov, V.A.; Tinyakov, P.G. Is the electric charge conserved in brane world? J. High Energy Phys. 2000, 8, 041. [CrossRef]

96. Dubovsky, S.L.; Rubakov, V.A. On models of gauge field localization on a brane. Int. J. Mod. Phys. A 2001, 16, 4331. [CrossRef] 
97. Dvali, G.; Gabadadze, G.; Shifman, M. (Quasi)Localized Gauge Field on a Brane: Dissipating Cosmic Radiation to Extra Dimensions? Phys. Lett. B 2001, 497, 271. [CrossRef]

98. Ziaeepour, H. Color Glass Condensate in Brane Models or Don't Ultra High Energy Cosmic Rays Probe 1015eV Scale ?. Mod. Phys. Lett. A 2005, 20, 419. [CrossRef]

99. Ziaeepour, H. QCD Color Glass Condensate Model in Warped Brane Models. Grav. Cosmol. Suppl. 2005, $11,189$.

100. Hong, D.-K.; Hsu, S.D.H. Holography, Entropy and Extra Dimensions. Phys. Lett. B 2004, 591, 208. [CrossRef]

101. Creek, S.; Gregory, R.; Kanti, P.; Mistry, B. Braneworld stars and black holes. Class. Quant. Grav. 2006, 23, 6633. [CrossRef]

102. CMS Collaboration. Search for microscopic black hole signatures at the Large Hadron Collider. Phys. Lett. B 2011, 697, 434. [CrossRef]

103. Dvali, G.; Gomez, C.; Mukhanov, S. Probing Quantum Geometry at LHC. J. High Energy Phys. 2011, $1102,12$. [CrossRef]

104. Dvali, G.; Folkerts, S.; Germani, C. Physics of Trans-Planckian Gravity. Phys. Rev. D 2011, 84, 024039. [CrossRef]

105. Dvali, G.; Giudice, G.F.; Gomez, C.; Kehagias, A. UV-Completion by Classicalization. J. High Energy Phys. 2011, 08, 108. [CrossRef]

106. Padmanabhan, T. Gravity and the Thermodynamics of Horizons. Phys. Rep. 2005, 406, 49. [CrossRef]

107. Verlinde, E.P. On the Origin of Gravity and the Laws of Newton. J. High Energy Phys. 2011, $1104,029$. [CrossRef]

108. Hu, B.L. Can Spacetime be a Condensate? Int. J. Theor. Phys. 2005, 44, 1785. [CrossRef]

109. Sindoni, L. Emergent gravitational dynamics from multi-BEC hydrodynamics?. Phys. Rev. D 2011, 83, 024022. [CrossRef]

110. 't Hooft, G. Dimensional Reduction in Quantum Gravity. arXiv 1993, arXiv:gr-qc/9310026.

111. Van Raamsdonk, M. Building up spacetime with quantum entanglement. Gen. Rel. Grav 2010, 42, $2323-2329$. [CrossRef]

112. Jacobson, T. Gravitation and vacuum entanglement entropy. Int. J. Mod. Phys. D 2012, 21, 1242006. [CrossRef]

113. Piazza, F. Glimmers of a pre-geometric perspective. Found. Phys. 2010, 40, 239. [CrossRef]

114. Eichhorn, A.; Koslowski, T.; Lumma, J.; Pereira, A.D. Towards background independent quantum gravity with tensor models. Class. Quant. Grav. 2019, 36, 15. [CrossRef]

115. Maldacena, J.M. The Large N Limit of Superconformal Field Theories and Supergravity. Adv. Theor. Math. Phys 1998, 2, 231. [CrossRef]

116. Donnelly, W.; Giddings, S.B. Diffeomorphism-invariant observables and their nonlocal algebra. Phys. Rev. D 2016, 93, 024030. [CrossRef]

117. Giddings, S.B. Quantum-first gravity. Found. Phys. 2019, 49, 177. [CrossRef]

118. Dirac, P.A.M. The Principles of Quantum Mechanics; Oxford University Press: Oxford, UK, 1958.

119. Von Neumann, J. Mathematical Foundation of Quantum Theory; Princeton University Press: Princeton, NJ, USA, 1955.

120. Kiefer, C. On the interpretation of quantum theory-from Copenhagen to the present day. In Time, Quantum and Information; Springer: Berlin/Heidelberg, Germany, 2003.

Publisher's Note: MDPI stays neutral with regard to jurisdictional claims in published maps and institutional affiliations.

(C) 2020 by the author. Licensee MDPI, Basel, Switzerland. This article is an open access article distributed under the terms and conditions of the Creative Commons Attribution (CC BY) license (http:/ / creativecommons.org/licenses/by/4.0/). 